\title{
Elemental Abundance Ratios in Stars of the Outer Galactic Disk. II. Field Red Giants ${ }^{1}$
}

\author{
Bruce W. Carney \\ Department of Physics \& Astronomy, University of North Carolina, Chapel Hill, NC \\ 27599-3255; email: bruce@physics.unc.edu \\ David Yong \\ Department of Physics $\&$ Astronomy, University of North Carolina, Chapel Hill, NC \\ 27599-3255; email: yong@physics.unc.edu \\ Maria Luísa Teixera de Almeida \\ Department of Physics 83 Astronomy, University of North Carolina, Chapel Hill, NC \\ 27599-3255; email: luisa@oal.ul.pt \\ Patrick Seitzer \\ Department of Astronomy, 818 Dennison Building, University of Michigan, Ann Arbor, MI \\ 48109; email: pseitzer@umich.edu
}

\begin{abstract}
We summarize a selection process to identify red giants in the direction of the southern warp of the Galactic disk, employing $V I_{C}$ photometry and multi-object spectroscopy. We also present results from follow-up high-resolution, high-S/N echelle spectroscopy of three field red giants, finding $[\mathrm{Fe} / \mathrm{H}]$ values of about -0.5 . The field stars, with Galactocentric distances estimated at 10 to $15 \mathrm{kpc}$, support the conclusion of Yong, Carney, \& de Almeida (2005) that the Galactic metallicity gradient disappears beyond $R_{\mathrm{GC}}$ values of 10 to $12 \mathrm{kpc}$ for the older stars and clusters of the outer disk. We summarize the detailed abundance patterns for 15 other elements for these stars and compare them to recently-obtained results for old open cluster red giants in the outer disk. The field and cluster stars at such large distances show very similar abundance patterns, and, in particular, all show enhancements of the " $\alpha$ " elements $\mathrm{O}, \mathrm{Mg}, \mathrm{Si}, \mathrm{Ca}$, and $\mathrm{Ti}$ and the $r$-process element Eu. These results suggest that Type II supernovae have been significant contributors to star formation in the outer disk relative to Type Ia supernovae within the past few Gyrs. We also compare our results with those available for
\end{abstract}


much younger objects. The limited results for the H II regions and B stars in the outer disk also suggest that the radial metallicity gradient in the outer disk is shallow or absent. The much more extensive results for Cepheids confirm these trends, and that the change in slope of the metallicity gradient may occur at a larger Galactocentric distance than for the older stars and clusters. However, the younger stars also show rising $\alpha$ element enhancements with increasing $R_{\mathrm{GC}}$, at least beyond $12 \mathrm{kpc}$. These trends are consistent with the idea of a progressive growth in the size of the Galactic disk with time, and episodic enrichment by Type II supernovae as part of the disk's growth.

Subject headings: Galaxy — disk; Clusters — abundances

\section{INTRODUCTION}

In this paper we continue our discussion of metallicities, $[\mathrm{Fe} / \mathrm{H}]$, and elemental abundance ratios, $[\mathrm{X} / \mathrm{Fe}]$, for stars in the outer Galactic disk. In Paper I (Yong, Carney, \& de Almeida 2005), we described a set of radial velocity measures and abundance determinations of four old open clusters whose Galactocentric distances, $R_{G C}$, lie between 12 and 23 kpc. Three stars in the local old open cluster M67 were observed and analyzed in the same fashion to provide checks on both the velocities and abundances.

Here we investigate a slightly different sample, three field red giants. Selecting distant field giants in the sea of foreground field stars, and in the presence of large and variable interstellar extinction, presents a challenge, but we have exploited a few key tricks. First, we believe that the distant outer disk is detectable, based on our earlier work in two key directions toward the southern hemisphere Galactic warp (Carney \& Seitzer 1993). Second, modulo typical disk population line-of-sight velocity dispersions $\left(\approx 20 \mathrm{~km} \mathrm{~s}^{-1}\right)$, disk stars obey a fairly clear rotation curve. Depending on the Galactic longitude, radial velocities of disk stars closer to and farther from the Sun will yield rather different radial velocities. We exploit this here by first obtaining extensive $V I_{C}$ photometry in the general direction of one of the fields studied by Carney \& Seitzer. From the color-magnitude diagram, we identified

\footnotetext{
${ }^{1}$ This paper makes use of obsevations obtained at the National Optical Astronomy Observatory, which is operated by AURA, Inc., under contract from the National Science Foundation. We also employ data products from the Two Micron All Sky Survey, which is a joint project of the University of Massachusetts and the Infrared Processing and Analysis Center/California Institute of Technology, funded by the National Aeronautics and Space Administration and the National Science Foundation
} 
likely red giants. We then obtained high-resolution, low signal-to-noise $(\mathrm{S} / \mathrm{N})$ spectra of over two hundred of these stars, and identified those whose radial velocities are most consistent with large Galactocentric distance. Three of these stars provide the program sample for this paper.

\section{OBSERVATIONS}

The disk of the Milky Way is warped, as seen using H I gas (Henderson et al. 1982; Burton \& Te Lintel Hekkert 1986; Diplas \& Savage 1991), CO emission from IRAS point sources (Wouterloot et al. 1990), and the dust layer, as seen by IRAS (Sodroski et al. 1987) and DIRBE (Freudenreich et al. 1994). Unlike many other disk galaxies, the Milky Way's warp appears to contain stars, including OB stars (Miyamoto et al. 1988; Orsatti 1992; Reed 1996), dusty-shell stars selected from the IRAS point source catalog (Djorgovski \& Sosin 1989), and K giants (López-Corredoira et al. 2002).

Detecting stars at large distances along lines of sight confined to the plane is nearly hopeless (except at infrared wavelength) due to interstellar extinction. Even methods that rely on gravity-sensitive intermediate-band photometric features, like the DDO-51 filter, are vulnerable to changes in reddening. But the warp offers us a possible means to peek above or below the Galactic murk and discern the most distant stars. Carney \& Seitzer (1993) detected excess numbers of stars along lines of sight to the southern Galactic warp compared to lines of sight at the same Galactic longitudes but opposite latitudes.

If we assume pure circular rotation for distant disk stars, the radial velocity of a star at Galactic longitude $(\ell)$ and latitude $(b)$ to be

$$
V_{L S R}=\left(\frac{\Theta}{R_{\mathrm{GC}}}-\frac{\Theta_{0}}{R_{0}}\right) R_{0} \sin \ell \cos b,
$$

where $R_{0}$ is the distance of the Sun from the Galactic center, $\Theta$ is the linear rotation speed of the Galactic disk at $\mathrm{r}=R_{G C}$, and $\Theta_{0}$ is the linear rotation speed at $R_{0}$. We adopt $R_{0}=$ $8 \mathrm{kpc}, \Theta_{0}=220 \mathrm{~km} \mathrm{~s}^{-1}$, and that the rotation curve beyond the solar circle is flat, so $\Theta$ is also $220 \mathrm{~km} \mathrm{~s}^{-1}$. Table 1 summarizes predicted purely circular, flat rotation curve values for $V_{L S R}$ as a function of $R_{G C}$, in the line of sight for one of the fields observed by Carney \& Seitzer (1993: their field "Warp 1a"), centered at $\ell=245.75$ and $b=-4.1$. Of course, we do not measure $V_{\mathrm{LSR}}$, but the heliocentric radial velocity, $V_{\text {rad }}$. The conversion of $V_{\text {rad }}$, to $V_{\mathrm{LSR}}$ was determined using Equation 2, where we assumed a Solar peculiar velocity, $V_{\mathrm{p}}$, of $20 \mathrm{~km} \mathrm{~s}^{-1}$ directed toward $\ell_{\mathrm{p}}=57$ and $b_{\mathrm{p}}=22$.

$$
V_{\mathrm{LSR}}=V_{\mathrm{rad}}+V_{\mathrm{p}} \times \frac{\left(\cos b_{\mathrm{p}} \cos \left(\ell-\ell_{\mathrm{p}}\right) \cos b+\sin b_{\mathrm{p}} \sin b\right)}{\cos b}
$$




\subsection{Photometry}

We observed Warp Field 1a with the University of Michigan's Curtis Schmidt telescope at Cerro Tololo Inter-American Observatory using a Thompson $1024 \times 1024$ Thompson CCD with an image scale of 1.835 arcsec per pixel and a field of view of $31^{\prime} \times 31^{\prime}$. The CCD was a thick frontside-illuminated device and coated to provide $U$ and $B$ sensitivity. We obtained images in $B, V$, and $I$, with exposure times ranging from 30 to 300 seconds. After using DAOPHOT to measure aperture magnitudes, we employed mean extinction coefficients and a set of 16 photometric standards from Graham (1982) to complete the transformation of the data to the standard Cousins photometric system. The scatter in the transformations was about $0.025 \mathrm{mag}$ per star, which is reasonably good considering the pixel scale of the data.

Table 2 summarizes our results for 8472 stars, and the resulting color-magnitude diagram is shown in Figure 1. It reveals a relatively typical Galactic disk population of field stars, and we assume that the stars with $12.5 \leq V \leq 17$ and $1.2 \leq V-I_{C} \leq 1.8$ are probably red giants lying over a wide (and indeterminate) range of distances. Our challenge was to select a few such stars for follow-up spectroscopic studies to determine $[\mathrm{Fe} / \mathrm{H}]$ and chemical abundance patterns, and we decided to employ Equation 1 to do so. Therefore, we needed to obtain radial velocities for a large number of those candidate red giants.

\subsection{Spectroscopic Observations: Multi-object Spectroscopy}

From the color-magnitude and color-diagrams constructed using our photometry, we selected stars for radial velocity measurements. Only stars redder than $V-I_{C}=+1.0$ were selected. The 24-fiber multi-object Argus echelle spectrograph was used at the prime focus of the Cerro Tololo Inter-American Observatory 4.0-m Blanco Telescope in March of 1994. Single-order spectra centered on the $\mathrm{Mg} b$ triplet $(\approx 5200 \AA$ ) were obtained for 209 of the stars that appear to be field red giants. We also obtained spectra for a number of well-observed red giants in the globular cluster $\omega$ Cen as well as bright radial velocity standards, HD 31871, HD 120223, and HD 176047. Radial velocities were determined using cross correlation techniques, and the typical velocity precision for our program stars is 2.5 $\mathrm{km} \mathrm{s}^{-1}$. Table 3 summarizes our results, along with $V I_{C}$ photometry from Table 2 , and $J H K$ photometry from $2 \mathrm{MASS}^{2}$. The positions have been taken from 2MASS.

\footnotetext{
${ }^{2}$ The Two Micron All-Sky Survey is a joint project of the University of Massachusetts and the Infrared Processing and Analysis Center/California Institute of Technology, funded by the National Aeronautics and Space Administration and the National Science Foundation.
} 


\subsection{Selection of Targets}

We selected three candidate red giants for further study. The red giant candidates in Figure 1 are faint, and we were restricted to the brighter stars. We therefore employed the radial velocities from Table 3 in comparison with the Galactic rotation model predictions from Table 1. All three stars have large radial velocities and relatively bright $V$ magnitudes. Figure 2 provides finding charts for the three stars.

\subsection{Spectroscopic Observations: Echelle}

The three targets were observed with the echelle spectrograph and the 4-meter telescope at the Cerro Tololo Inter-American Observatory (CTIO) during January 1998 and 1999. We employed the long red camera and the 31.6 lines $\mathrm{mm}^{-1}$ echelle grating. A GG495 filter blocked second-order blue light, while the G181 cross disperser (316 lines $\mathrm{mm}^{-1}$ ) led to a wavelength coverage of 5200-7940 $\AA$. The slit was opened to 150 microns, providing a width of $1.0^{\prime \prime}$ on the sky, yielding a spectral resolving power of 28,000 and a dispersion of 0.07 $\AA$ per pixel at $5800 \AA$, and providing two pixels per resolution element. Our goal was to derive accurate metallicities, $[\mathrm{Fe} / \mathrm{H}]$, as well as detailed element-to-iron abundance ratios to explore the chemical evolution of outer disk stars, following the same goals as presented in our previous paper (Yong et al. 2005).

The observing routine included 20 quartz lamp exposures to provide data for flatfielding, and 15 zero-second exposures (to provide "bias" frames). Th-Ar hollow cathode lamp spectra were taken before and after each stellar exposure. For radial velocity standards, we used the sky, and also HD 80170, which is a K giant, and similar in temperature and gravity to our program stars.

The spectroscopic data were reduced using IRAF $^{3}$ IMRED, CCDRED, and ECHELLE packages to correct for the bias level, trim the overscan region, extract individual orders, fit the continuum, apply a wavelength solution using the Th-Ar spectra (and determine a systematic correction using the observed radial velocity standard). Master flat field frames were produced each night, and normalized using APFLATTEN, following which the data frames were divided by the master flat field frames prior to extraction of individual orders using APALL.

\footnotetext{
${ }^{3}$ IRAF (Image Reduction and Analysis Facility) is distributed by the National Optical Astronomy Observatory, which is operated by the Association of Universities for Research in Astronomy, Inc., under contract with the National Science Foundation.
} 
The Th-Ar comparison spectra obtained before and after each program star exposure were used to measure radial velocities. ECIDENTIFY and DISPCOR were used to identify the lines and determine the dispersion solution for each order, and CONTINUUM task enabled us to interactively fit a high-order cubic spline to produce the continuum-normalized, wavelength-calibrated spectra. Stars with more than one observed spectrum were crosscorrelated and then combined into a single final spectrum using SCOMBINE. Table 4 includes the Heliocentric Julian Date of mid-exposure as well as the final S/N levels per pixel of the combined spectra.

\subsection{Echelle Radial Velocities}

We used Fourier cross-correlation techniques to derive radial velocities from our echelle spectra, employing FXCOR within the RV package in IRAF. Radial velocities were determined for many individual orders after rebinning to common log-linear dispersions, the edges of each order $(\approx 200$ pixels) were set to zero, and the edges, $\approx 0.2$ points, were apodized with a cosine bell curve. The maximum peak of the Fourier transform was fitted by a Gaussian, with its center used to determine the radial velocity. The observed radial velocities were then transformed into Heliocentric radial velocities using FXCOR. Table 4 includes the final results, the number of orders used, and the standard error per order.

For the three program stars, the agreement with the results from our Argus observations is very good, with a mean difference (echelle - Argus) of $+1.3 \mathrm{~km} \mathrm{~s}^{-1}$ and $\sigma=1.9 \mathrm{~km} \mathrm{~s}^{-1}$.

\section{ELEMENTAL ABUNDANCES}

\subsection{Stellar Parameters: Initial Estimates}

It is somewhat challenging to regard a line-rich high-resolution spectrum and make a good initial guess for the stellar parameters. Photometry is often invoked for such estimates, but the low Galactic latitude of our program stars also introduces difficulties. A good initial guess may be made in the following manner, however.

We first adopt a reddening estimate based on Schlegel, Finkbeiner, \& Davis (1998). The measured radial velocities and the results in Table 1 suggest that the stars lie at large distances, so we adopt a low metallicity, $[\mathrm{Fe} / \mathrm{H}] \approx-0.6$, based on the results from Paper I that found such low metallicities for old open clusters at essentially all Galactocentric distances greater than about $12 \mathrm{kpc}$. Temperatures and gravities may be estimated using 
the color-temperature and color-bolometric correction calibrations of Alonso, Arribas, and Martinez Roger (1999). The 2MASS photometry was transformed to the relevant IR system employed by Alonso et al. (1999) using the equations given by Alonso, Arribas, \& Martinez Roger (1994), and Carpenter (2001). We adopted masses of $1.0 M_{\odot}$ and the luminosities were derived using distances estimated (naively) from the measured radial velocities. Initial parameters for all three stars are given in Table 5.

\subsection{Refining the Stellar Parameters}

In Paper I, we demonstrated that our adopted atomic line parameters and methods of analysis resulted in good agreement between our derived abundances and published values for the Sun, Arcturus, and extensive prior work on the giants in the old open cluster M67. We refer the reader to that paper for a more complete discussion of our procedures, and of the sensitivity of our final abundances to our adopted values for $T_{\text {eff }}, \log g$, and $[\mathrm{Fe} / \mathrm{H}]$. In

brief, to derive the stellar parameters, we measured equivalent widths of Fe I and Fe II lines in our spectra. Table 6 provides the basic data.

We first determined $T_{\text {eff }}$ by using the weaker Fe I lines so that no trend between the derived iron abundance and lines' excitation potentials was present. We then included the stronger Fe I lines and adopted a microturbulent velocity, $V_{\text {turb }}$, such that the derived iron abundance was independent of equivalent width (and hence whether saturation of the line was present or not). The gravity was the final parameter determined, exploiting the requirement that the pressure-insensitive Fe I lines yield the same iron abundance as the pressure-sensitive Fe II lines. The final $[\mathrm{Fe} / \mathrm{H}]$ abundances are given in Table 7.

The adopted spectroscopic parameters differ somewhat from our initial estimates, but considering the difficulties involved, the agreement is quite good. The reddening estimates from Schlegel et al. (1998) are high, and hence somewhat uncertain, and of course they refer to the total along the line of sight, and over-estimate the true reddening and extinction for stars closer to the Sun. The initial gravity estimates are most sensitive to our initial assumptions about the stars' distances, which we determine on the basis of measured radial velocities and a radial velocity vs. distance relation that does not account for velocity dispersion nor the presence of the Galactic warp. 


\subsection{Other Elemental Abundances}

We derived abundances for fifteen other elements, using lines and $g f$ values provided in Table 6. For all fifteen elements, we relied on spectrum synthesis to derive the elemental abundances for each line. MOOG was employed to generate $8 \AA$ windows centered on each line and the elemental abundances were adjusted until the fit was judged to be optimal. A few elements required special attention. For Mn, we had to include the effects of hyperfine splitting, following Prochaska \& McWilliam (2000). In a number of cases, we also had to consider isotopic splitting, in which case we adopted solar isotopic abundance ratios. The elements so considered included Co and Ba (Prochaska et al. 2000), Rb (Lambert \& Luck 1976; Tomkin \& Lambert 1999), La (Lawler et al. 2001a), and Eu (Lawler et al. 2001b).

Final abundances are given for our three outer disk field stars in Table 7.

\section{DISCUSSION}

\subsection{Distance Estimations}

We are now in a position to substantially improve our distance estimates for the three program stars.

Our basic approach is as follows. First, we estimate the interstellar reddening to each star by requiring that the de-reddened $V-K$ color yield the spectroscopically-determined effective temperature using the color-temperature relations from Alonso et al. (1999). This leads to $\mathrm{E}(V-K)$ and $\mathrm{E}(B-V)(=\mathrm{E}(V-K) / 2.74)$. We are then able to determine $K_{0}$ and we then estimate $M_{K, 0}$, and, hence $(m-M)_{0}$, by referring to a cluster with known reddening and distance and with a similar metallicity as our program stars.

We have chosen Be 29 as our reference cluster for four reasons. First, it has a very similar metallicity as our three field stars, $[\mathrm{Fe} / \mathrm{H}]=-0.54$ according to Paper $\mathrm{I}$. The second advantage is that the cluster's iron abundance was determined using exactly the same observational and analysis procedures as employed for the field stars. Third, its reddening is quite low, estimated to be $\mathrm{E}(B-V) \approx 0.04$ in Paper I. Finally, it has reasonably well-defined optical and infrared color-magnitude diagrams. We prefer to work with infrared magnitudes because of their reduced sensitivity to extinction.

Before we undertake the comparisons, however, we apply the same reddening estimation procedure outlined above to the cluster. We are able to reproduce the temperatures and gravities of stars 988 and 673 we derived spectroscopically in Paper I if $\mathrm{E}(B-V)=0.16$ 
mag, and for a cluster distance modulus of 15.76 mag.

With the cluster's de-reddened $K_{0}$ vs. $T_{\text {eff }}$ diagram, and our estimated temperatures for the three field stars, we derive de-reddened $M_{K}$ values, distance moduli, heliocentric and Galactocentric distances, all of which are provided in Table 8. For star 9060, the estimate of $M_{K}$ is especially straightforward since its temperature and surface gravity indicate it is a red clump giant, and as discussed in Paper I, such stars form the basis for all of our distance estimates for the clusters. We have adopted the recommendation of Alves (2000) that $M_{K}$ $=-1.61 \mathrm{mag}$ for such stars.

\subsection{Comparison with Old Open Clusters}

\subsubsection{Differences in Elemental Abundance Patterns}

Although it appears that the field stars lie at large Galactocentric distances, their ages are unknown. The first question we ask is whether the chemical abundances of these field stars resemble those of the outer disk old open clusters. We approach this question first by noting that all three field stars have very similar abundance patterns, and we therefore average the element-to-iron abundance ratios for all three stars. We do the same for the old open clusters studied in Paper I, averaging the results for all six stars, except for the neutron capture elements. In Paper I we noted that two of the clusters, Be 31 and NGC 2141, show significantly elevated abundances of the light $s$-process element $\mathrm{Zr}$, the heavy $s$-process elements $\mathrm{Ba}$ and $\mathrm{La}$, and the $r$-process element $\mathrm{Eu}$. We therefore have computed three sets of averages for these elements: all six stars in the four outer disk clusters, the four stars in Be 20 and Be 29, and the two stars in Be 31 and NGC 2141. Finally, for comparison purposes, we have also averaged the results for the three stars we studied in the old open cluster M67. Our results are summarized in Table 9 and Figure 3. We remind the reader that all of these stars were observed with similar equipment and analyzed together, so while systematic errors in the analyses may compromise comparisons with results of other workers, our comparisons between the field stars, cluster stars, and the Sun should be robust.

Perhaps the first result of note is that the old open clusters and the field stars in the outer disk are essentially identical in their $[\mathrm{X} / \mathrm{Fe}]$ abundance distributions. The only significant differences are in the neutron-capture elements. If we restrict the comparison of the field stars to the four stars we studied in Be 20 and Be 29, we find that the field stars are very similar to the cluster stars in their mean La abundances, but the field stars have lower $\mathrm{Ba}$ abundances and somewhat higher Eu abundances.

Let us now make comparisons that include the comparable age but more metal-rich and 
smaller Galactocentric distance cluster M67.

The light elements $\mathrm{Na}$ and $\mathrm{Al}$ are similar in all three sets of stars: the field stars and the stars in the comparable age clusters of the outer disk and M67. There are minor and probably insignificant differences in the iron peak elements $\mathrm{Co}$ and $\mathrm{Ni}$, and $\mathrm{Mn}$ may be more deficient relative to iron in the more metal-poor stars of the outer disk.

The most notable differences between the outer disk field and cluster stars and those of M67 and the Sun are in the " $\alpha$ " elements, O, Mg, Si, Ca, and Ti, and the $r$-process element Eu. In the field stars, as in the old open clusters of the outer disk, the $[\alpha / \mathrm{Fe}]$ and $[\mathrm{Eu} / \mathrm{Fe}]$ abundance ratios are enhanced.

In summary, the field stars appear to behave very similarly to the stars in the old open clusters at comparable Galactocentric distances. This has an interesting consequence regarding the origins of these field stars. One objection to our finding of elevated $[\alpha / \mathrm{Fe}]$ ratios in outer disk stars is that such behavior is often seen in stars belonging to the Galactic thick disk (Fuhrmann 1998; Prochaska et al. 2000; Bensby et al. 2003, 2004; Brewer \& Carney 2004, 2005). Even at the low latitudes at which we are looking, our three stars have estimated distances from the Galactic plane of about 300 to 650 pc, far enough to normally qualify for thick disk membership. However, the thick disk appears to be uniformly old (Bensby et al. 2004; Brewer \& Carney 2004, 2005), and the ages of the old open clusters are not consistent with thick disk membership. The very close agreement between the three field stars studied here and the old open clusters suggests similar origins, from which we conclude that the field stars are also unlikely to belong to the thick disk.

We now consider what the two sets of stars reveal about chemical evolution in the outer Galactic disk.

\subsubsection{Trends with Galactocentric Distance}

In Paper I we drew attention to the apparent disappearance of the Galactic metallicity gradient for $R_{\mathrm{GC}}>12 \mathrm{kpc}$. The three field stars confirm this behavior, and, in fact, appear to show that the radial abundance gradient for older stars and clusters may disappear at an even smaller Galactocentric distance, perhaps $10 \mathrm{kpc}$. This result is hard to understand in simple models of Galactic chemical evolution. A metallicity gradient arises in the chemical evolution of a closed system, with lower-density outer regions evolving more slowly than the higher-density inner regions. At any epoch, the transformation of gas into stars should be less complete in the outer regions, and the resultant mean metallicity should be lower. But what if the Galaxy is not a closed system? Twarog, Ashman, \& Anthony-Twarog (1997) 
addressed this question in their study of the mean metallicities of a large sample of open clusters. Twarog et al. (1997) noticed that the clusters also showed a break in the metallicity gradient at $R_{\mathrm{GC}} \approx 10 \mathrm{kpc}$, with an essentially constant value at larger Galactocentric distance $^{4}$. Twarog et al. (1997) considered this result and offered what we believe to be a viable explanation. If the outer disk is the repository of a considerable amount of gas accreted by the Galaxy's disk over a long period of time, a metallicity gradient might not be expected. Indeed, Twarog et al. (1997) speculated that the transition zone, at $R_{\mathrm{GC}} \approx 10$ kpc, may represent the boundary of the "original" Galactic disk.

Of course, a key question is whether additional data further support the suggestions of Twarog et al. (1997). Chen, Hou, \& Wang (2003) suggested that the discontinuity seen by Twarog et al. (1997) is not apparent in their study. Their analyses do show that a linear fit to the radial metallicity gradient does appear to be steeper for older clusters. Unfortunately, they do not cite the uncertainties of the derived metallicity gradients, nor attempt any nonlinear fits that our data indicate should be undertaken. Perhaps the best that can be said at this point, in our opinion, is that the plotted data of Chen et al. (2003) are consistent with the results of Twarog et al. (1997) and our own work in Paper I.

Our results pose two additional difficulties. First, our results and those of Twarog et al. (1997) suggest that there does not appear to be a significant dispersion in $[\mathrm{Fe} / \mathrm{H}]$ at large Galactocentric distances. Not only is there little to no metallicity gradient, but the three field stars and three of the four outer disk open clusters we analyzed in Paper I show very similar $[\mathrm{Fe} / \mathrm{H}]$ values. If we include the three clusters from Paper I with $R_{\mathrm{GC}}>12 \mathrm{kpc}$, Be $20\left(R_{\mathrm{GC}}=16.0 \mathrm{kpc} ;[\mathrm{Fe} / \mathrm{H}]=-0.45\right)$, Be $29\left(R_{\mathrm{GC}}=22.5 \mathrm{kpc} ;[\mathrm{Fe} / \mathrm{H}]=-0.54\right)$, and Be $31\left(R_{\mathrm{GC}}=12.9 \mathrm{kpc},[\mathrm{Fe} / \mathrm{H}]=-0.57\right)$, and the three field stars, we find $\left.<[\mathrm{Fe} / \mathrm{H}]\right\rangle=$ -0.48 , with a standard error of only 0.07 dex, which is probably the relative uncertainty of the individual measures.

We can not offer an explanation for the very similar metallicities, but draw attention to it as a feature that must be explained along with the absence of a metallicity gradient. Of course, our sample is small, and so we might be vulnerable to the vagaries of statistical chance. However, the much larger number of photometric metallicity estimates that form the basis of the results of Twarog et al. (1997) suggest similar behavior.

The other puzzle, and to which we drew special attention in Paper I, and which recurs with the field stars, is the near-uniform enhancement of the $\alpha$ elements, including oxygen.

\footnotetext{
${ }^{4}$ It is interesting that Janes (1979) had also seen similar behavior in his Figure 10, but, unfortunately, his Figure 11 binned the data and erased the change in the metallicity gradient, supplanting it with an apparently even steeper one!
} 
In Figure 4 we show this graphically, using the averaged abundances of $\mathrm{Mg}, \mathrm{Si}, \mathrm{Ca}$, and $\mathrm{Ti}$ with respect to iron, plotted vs. Galactocentric distance. The differences with respect to M67 are modest, somewhat over 0.1 dex, but relative to the Sun, whose age is comparable to M67 and the outer disk clusters, the difference is remarkable, amounting to roughly 0.2 dex. As we discussed in Paper I, it is extremely difficult to understand enhancements of these elements, and of Eu, within closed box models. One expects such enhancements in stars that formed shortly after star formation commenced, as is seen in the metal-poor and very old stars of the Galactic halo and thick disk, because at that point ejecta from Type II supernovae were dominating the heavy element enrichment of the interstellar medium. Solar $[\alpha / \mathrm{Fe}]$ ratios are achieved at a much later time, once Type Ia supernovae have had sufficient time (perhaps one to a few billion years) to contribute ejecta more enriched in iron than in the $\alpha$ or $r$-process elements. The problem for the clusters, and, we assume, for the field stars in the outer disk is that the old open clusters are not as old as the halo or metal-poor stars of the thick disk, but have ages of less than 6 Gyrs, comparable to that of the Sun. If SNe II have played a major role in the nucleosynthetic history of the outer disk, they have done so relatively recently, and not long before these clusters (and field stars) formed. SNe Ia have contributed as well, since the $[\alpha / \mathrm{Fe}]$ and $[\mathrm{Eu} / \mathrm{Fe}]$ are not as high as are found in the Galactic halo, and in Paper I we noted that AGB stars have also contributed to the interstellar medium, judging by the significant abundances of elements largely produced by $s$-process nucleosynthesis. (The same must be true for our field stars since they share similar abundance patterns as the cluster stars.) One way to explain these observations is to assume that the growth of the Galactic disk has been intermittent, and that merger events, be they large or small, have triggered episodes of star formation so that SNe II have been able to contribute anew to the chemistry of the interstellar medium, unlike the case in the Solar neighborhood, where $[\alpha / \mathrm{Fe}]$ and $[\mathrm{Eu} / \mathrm{Fe}]$ are, essentially, solar. The star formation may have continued long enough to enable the interstellar medium to have become enriched by AGB stars and also Type Ia supernovae, but not to the same degree as are seen in the thin disk stars of the solar neighborhood. We look forward to chemical evolution models that may help explain our observations.

Let us consider the "growth of the Galactic disk" and merger models a bit further. In Paper I we compared the velocities of the old open clusters with the "GASS" (Global Anticenter Stellar Structure) discussed by Frinchaboy et al. (2004), which may be related to the Monoceros Ring (Newburg et al. 2002; Ibata et al. 2003; Rocha-Pinto et al. 2003; Yanny et al. 2003) and the possible "Canis Major galaxy" (Martin et al. 2004). Table 4 includes the "Galactic System Reference" velocity, $V_{\mathrm{GSR}}$. Comparison with Figure 2 of Frinchaboy et al. (2004) indicates that all three field stars have velocities consistent with membership in the putative GASS. We remind the reader that GASS does not differ markedly 
in inclination from the Galactic disk ( $\approx 17$ degrees according to Frinchaboy et al. $2004 ; 25 \pm 5$ degrees, according to Peñarrubia et al. 2004) and since both respond to the same Galactic gravitational potential, radial velocities may not provide a particularly good discriminant regarding membership.

If the old open clusters in the outer disk or the three field stars studied here are to be associated with the GASS, there is a curious difference in the mean metallicities and metallicity spreads. Yanny et al. (2003) suggested the the mean $[\mathrm{Fe} / \mathrm{H}]$ value for the stars in the Monoceros Ring, which is thought to be related to the GASS, is near -1.6, much more metal-poor than we have found in the outer disk stars and clusters. The study of field M giants to identify the Monoceros Ring by Rocha-Pinto et al. (2003) and Crane et al. (2003) suggests that metal-rich stars may also be part of the GASS, and therefore that the metallicity range in the GASS may be very large. This is inconsistent with what we have found among our limited study of outer disk clusters and stars. Of course, the simplest explanation is that the objects we have studied do not belong to the GASS, but are representative of the Galactic outer disk.

The similar $[\mathrm{Fe} / \mathrm{H}]$ and $[\mathrm{X} / \mathrm{Fe}]$ patterns seen among the three field stars indicates similar chemical enrichment histories, but we note again that the enhancements of $[\alpha / \mathrm{Fe}]$ are identical in Be 29 (a GASS member) and Be 31 (a non-member). Further, the globular cluster NGC 2298 has been suggested to be a member of GASS, and it shows similar elevated $[\alpha / \mathrm{Fe}]$ values despite being several Gyrs older than any of the open clusters we have studied. We have invoked the idea of episodic star formation to explain enhancements of $[\alpha / \mathrm{Fe}]$ seen in the younger open clusters, but do we expect this to happen in small galaxies which are ultimately captured by the Milky Way? Or is it more likely that the triggered star formation happens when the smaller galaxy finally merges with the Milky Way? We believe the second option is the more viable explanation. As Venn et al. (2004) have discussed, the current dwarf galaxies surrounding the Milky Way show solar (and even sub-solar) $[\alpha / \mathrm{Fe}]$ abundance ratios and at very low metallicities. It would be surprising if the recently merged or merging dwarf galaxy that contains NGC 2298 and a host of younger and more metal-rich open clusters would deviate from this trend.

An attractive alternate view, in our opinion, is that the outer disk has experienced a number of smaller accretion events, involving metal-poor gas, and which triggered bursts of star formation so that SNe II helped rapidly enrich the interstellar medium and produce the enhanced abundances of $\alpha$ and $r$-process elements. If the Canis Major galaxy is a real structure (and our three field stars lie very near the direction to that galaxy's center, according to Martin et al. 2004), this may only be the latest such accretion event. In this view, the Galaxy's disk has grown steadily with time, as Twarog et al. (1997) described. 
One way to explore this further, perhaps, is to consider stars that have formed more recently, out of the more recently enlarged Galactic disk.

\subsection{Comparison with Young Stars \& Nebulae: Cepheids}

Paper I concentrated on the older known clusters with large Galactocentric distances, and in this paper we have considered field stars with unknown but probably large, ages.

We concentrate on the extensive and thorough abundance analyses of Cepheid variable stars undertaken by Andrievsky et al. (2002a,b,c; 2003) and Luck et al. (2003). In Figure 5 we repeat the results for older open clusters in the outer Galactic disk, the three field stars from this paper, and all the Cepheids studied, except that we eliminated redundant listings of stars, and retained only those stars for which the abundances of $\mathrm{Mg}, \mathrm{Si}, \mathrm{Ca}$, and $\mathrm{Ti}$ are available from these papers.

We focus first on the behavior of $[\mathrm{Fe} / \mathrm{H}]$ vs. $R_{\mathrm{GC}}$. There are three notable points in Figure 5. First, the Cepheids appear to show a metallicity gradient, with the lowest value reached for EE Mon at $R_{\mathrm{GC}} \approx 15.1 \mathrm{kpc}$. Second, the gradient may not be as simple as a linear decline in $[\mathrm{Fe} / \mathrm{H}]$ with $R_{\mathrm{GC}}$. One may argue that beyond about $12 \mathrm{kpc}$, there is no real gradient, if one ignores EE Mon. Andrievsky et al. (2002c), Luck et al. (2003), and Andrievsky et al. (2003) argued that the change in $[\mathrm{Fe} / \mathrm{H}]$ trends (or lack thereof) beyond $R_{\mathrm{GC}} \approx 10 \mathrm{kpc}$ may be due the effects of the Galaxy's co-rotation point, where the spiral arm pattern speed matches the stellar circular speeds. We argued in Paper I that while this model has some merit, it does not seem capable of explaining the same lack of a metallicity gradient in the old open clusters (and now seen as well in the three field stars) because the behavior extends over much larger Galactocentric distances, from 10 to over $20 \mathrm{kpc}$. Let us consider, then, that the metallicity gradient seen in the Cepheids is real, and that it reaches a fairly low metallicity, apparently $[\mathrm{Fe} / \mathrm{H}] \approx-0.2$, at $R_{\mathrm{GC}} \approx 12 \mathrm{kpc}$. (It may yet drop again, based on the most distant star, EE Mon.) If this version of the Cepheid radial metallicity gradient is correct, it implies that the younger stars show the same behavior as the older stars, but the metallicity trend is displaced to larger Galactocentric distances, and perhaps to high metallicities. This is consistent with the idea that the disk has grown radially over time, and that its mean metallicity has risen as well (but, again, recall the result for EE Mon).

Figure 5 also shows the trend in the alpha elements' abundances relative to iron, as a function of $R_{\mathrm{GC}}$. The bulk of the cepheids (again excluding EE Mon) show an essentially constant value for $[\alpha / H]$, consistent with no metallicity gradient. Since comparisons of 
metallicity gradients often compare results for iron from studies of clusters with abundances of the lighter $\alpha$ elements from abundances of H II regions, it is worth keeping in mind the different nucleosynthetic origins of these elements, and making direct comparisons of elements created in the same processes.

We turn now to the question of enhanced abundances of elements manufactured in SNe II, relative to those produced in SNe Ia, in particular $[\alpha / \mathrm{Fe}]$ vs. $R_{\mathrm{GC}}$. Figure 5 shows that $[\alpha / \mathrm{Fe}]$ in the Cepheids increases at larger Galactocentric distances, beyond about $12 \mathrm{kpc}$, and that this rise is similar in slope to that seen in the older stars and clusters, except that the rise begins at a somewhat larger Galactocentric distance in the younger Cepheids than in the older clusters and field stars. This is also consistent with the idea that the disk is continuing to grow, but that the effects of star formation triggered by infall are most pronounced at Galactocentric distances of $15 \mathrm{kpc}$ (and perhaps beyond?) rather than at $10 \mathrm{kpc}$ as was the case in the past. Again, this is qualitatively consistent with a growing Galactic disk. (And it is hard to explain in terms of the co-rotation point model of Luck et al. 2003 and Andrievsky et al. 2003.) We need careful (and consistent!) abundance analyses of the few Cepheids with $R_{\mathrm{GC}}$ values of $15 \mathrm{kpc}$ and larger to explore these trends further.

In a future paper we will present results for a small sample of distant but very young and easily studied Cepheid variables of the outer disk. It is, nonetheless, worthwhile to consider what is available already in the literature.

\subsection{Comparison with Young Stars \& Nebulae: B Stars \& H II Regions}

Cepheids have several advantages, being cool enough to provide absorption lines for many elements, and such stars also have well-determined distances. Cepheids have one problem, however, in that mixing may alter the abundances of some key elements, such as carbon, nitrogen, and oxygen. It is therefore worth exploring the Galactic disk's metallicity gradient using other tracers of recent star formation.

Shaver et al. (1983) provided a comprehensive study of Galactic H II regions whose $R_{\mathrm{GC}}$ values ranged from about 4 to almost $14 \mathrm{kpc}$, and Fich \& Silkey (1991) extended the work to roughly $18 \mathrm{kpc}$. While the scatter for any one measurement is relatively large, the data are consistent with what is seen for the Cepheids: the oxygen abundances reach a basement value (as do those of sulfur and nitrogen) at between 10 and $12 \mathrm{kpc}$. More recent work by Vílchez \& Esteban (1996) shows that the five H II regions they analyzed with $R_{\mathrm{GC}}>15 \mathrm{kpc}$ have very similar oxygen abundances, consistent with the trend seen for the $\alpha$ elements seen by Fich \& Silkey (1991) and in Figure 5. Henry \& Worthey (1999) have provided 
a good summary of all published work, and these trends are confirmed, although there is considerable scatter in the oxygen abundances at any Galactocentric distance. It is unclear if the scatter seen is due to true changes in metallicity or systematics of the analyses. The approximate constant, but lower, abundances of oxygen seen in H II regions beyond $15 \mathrm{kpc}$ or so is intriguing, and it is therefore even more important to push the study of $[\mathrm{Fe} / \mathrm{H}]$ and $[\alpha / \mathrm{Fe}]$ to such large distances using such tracers.

Rolleston et al. (2000) studied abundances in 80 B stars in 19 young open clusters, about half of which have $R_{G C}>10 \mathrm{kpc}$. They considered the suggestion by Twarog et al. (1997) that a step function exists in the Galactic metallicity gradient. They found that magnesium abundances appear to be independent of $R_{\mathrm{GC}}$, at least for distances outside 10 to $12 \mathrm{kpc}$, which is consistent with Figure 5. However, two of the other $\alpha$ elements, oxygen and silicon, do not show as clear a trend, but considerable scatter at distances beyond $12 \mathrm{kpc}$.

More recently, Daflon \& Cunha (2004) have provided a very careful exploration of all available Galactic B star data, seeking signs of differences as a function of $T_{\text {eff }}$ of the program stars or their rotational velocities, finding no significant effects among the stars they studied. Further, most of the elements studied behave similarly, which means that we may explore a mean $\alpha$ element abundance as a function of $R_{\mathrm{GC}}$. (Unfortunately, iron abundances are not available for these stars.) We adopt their abundances for oxygen, magnesium, silicon, and sulfur, and then correct them relative to solar photospheric abundances taken from Grevesse \& Sauval (1998). We take straight unweighted means of these four elemental abundances, discarding any star for which the abundances for the elements is incomplete. Figure 6 summarizes the inner and outer disk abundances. While it is certainly possible to draw a straight line fit, we assert that the data are also consistent, within the uncertainties, with the results of the Cepheids seen in Figure 5.

\subsection{Comparison with Other Galaxies}

Is our Galaxy special or do similar transitions between well-defined metallicity gradients and their apparent disappearance at some transition radius occur in other disk galaxies? An exhaustive review of extra-galactic metallicity gradients is inappropriate in this paper, but we draw attention to some relevant points.

Metallicity gradients have routinely been discussed in linear terms, either changes in logarithmic abundance vs. radial distance (Pagel \& Edmunds 1981) or fractional isophotal diameter (Zaritsky, Kennicutt, \& Huchra 1994). Our point, that linear trends may not be applicable to the total extent of our Galaxy's disk, appears to also be the case in at least 
some other galaxies. Zaritsky et al. (1994), in particular, questioned whether exponential behavior might be more descriptive for some galaxies. We speculate that if accretion is the cause for the lack of a gradient in our Galaxy's outer disk, it would be worth studying galaxies showing similar behavior to ascertain if such a characteristic is related to some other property, such as cluster environment, or, possibly, galactic mass. We suggest that galaxies in denser environments or with larger masses will have more opportunities for accretion events to alter the metallicity gradients in the outer disks.

Metallicity gradients alone will not provide the insight we seek, however. The nucleosynthetic history is revealed in element-to-iron ratios, and iron abundances are rarely obtained in the studies of H II regions, which provide the bulk of the results in the studies of other galaxies. We choose to concentrate on the most obvious comparison galaxy, M31 (= NGC 224). Here, at least, we have more detailed elemental abundances available for the $\mathrm{H}$ II regions and also a limited amount of information from abundance analyses of individual stars, including A supergiants (Venn et al. 2000) and B supergiants (Trundle et al. 2002). Like H II regions, these stars represent a snapshot of the recent star formation history in the disk of M31. Abundances of $\alpha$ elements in older disk stars, represented by normal K giants, are not available, and abundances of planetary nebulae outside the bulge of M31 (see Jacoby \& Ciardullo 1999) are very few in number.

We begin by considering the metallicity gradient, represented by the element oxygen. Figure 7 shows the logarithmic abundances of oxygen from studies of H II regions (Dennefeld \& Kunth 1981; Blair, Kirshner, \& Chevalier 1982) and the stars studied by Venn et al. (2000) and Trundle et al. (2002). We again transform logarithmic abundances relative to hydrogen to values relative to the Sun using the solar abundances from Grevesse \& Sauval (1998). Taking all $17 \mathrm{H}$ II regions, there is a detectable metallicity gradient, $\Delta[O / H]=$ $-0.028 \pm 0.007 \mathrm{dex} \mathrm{kpc}^{-1}$. Adding in the ten stars yields $\Delta[O / H]=-0.017 \pm 0.008$ dex $\mathrm{kpc}^{-1}$. But M31's young disk population, like the old population of the Milky Way, appears to show a shallower, or even absent, metallicity gradient beyond a distance of about $10 \mathrm{kpc}$.

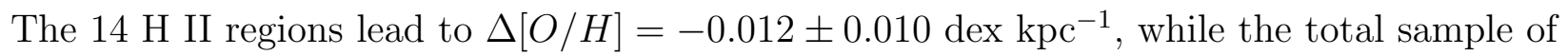
$17 \mathrm{H}$ II regions and stars yields $\Delta[O / H]=-0.006 \pm 0.008 \mathrm{dex} \mathrm{kpc}^{-1}$.

The stellar abundances provide one more interesting piece of evidence. Figure 8 presents the very limited data regarding the relative contributions of SNe II, represented by oxygen, relative to the contributions of SNe Ia, represented by iron. A single datum with enhanced $[\mathrm{O} / \mathrm{Fe}]$ at large galactocentric distance hardly proves the similarity of M31 to the Galaxy, but at least it does point the way to future work. 


\section{CONCLUSIONS}

We have used color-magnitude diagrams and radial velocities obtained from multi-fiber spectroscopy to identify candidate red giants of the outer Galactic disk. High-resolution, high-S/N echelle spectrscopy has confirmed these identifications for three stars lying in the direction of the southern Galactic warp. The $[\mathrm{Fe} / \mathrm{H}]$ values and Galactocentric distances of these stars confirms the results found in Paper I. At Galactocentric distances beyond 10 to 12 kpc, the Galactic metallicity gradient for the moderately old stars and clusters disappears. Further, abundances of elements manufactured primarily in SNe II are enhanced in both the field stars and the old open clusters of the outer Galactic disk, despite the fact that none of the clusters have ages greater than about 6 Gyrs. In the solar neighborhood, abundances of the elements produced largely in SNe II are much lower in stars and clusters with these ages, showing the outer disk has experienced a very different star formation history. We suggest that the difference is caused by a gradual growth of the outer disk. Episodic accretion can erase a metallicity gradient, and if the accretion events trigger bursts of star formation over a period of time, it may also explain the elevated abundances of the $\alpha$ elements and the $r$ process element Eu. The available data for the much younger Cepheids suggest similar trends may be at work there, too, but, in essence, displaced to larger Galactocentric distances. H II regions and $\mathrm{B}$ stars appear to support the idea of a reduced or absent metallicity gradient among young objects in the outer disk. This, too, is consistent with the steady growth and episodic chemical enrichment of the outer disk. The limited data for young stars and H II regions in the disk of M31 hint at similar behavior.

Future measurements of iron and $\alpha$ element abundances are certainly desirable for more stars across the disk of M31, as well as for M33, and for both younger and older stars in the outer disk of the Milky Way. Cepheid variables with $R_{\mathrm{GC}} \geq 15 \mathrm{kpc}$ would be most welcome, as would abundance of objects more clearly associated with the Monoceros Ring and other star streams.

We are extremely grateful to the National Science Foundation for their financial support

through grants AST-9619381, AST-9988156, and AST-0305431 to the University of North Carolina. 
Table 1. Predicted radial velocities relative to the Local Standard of Rest for different Galactocentric distances toward $\ell=245.75, b=-4.1$.

\begin{tabular}{rrrr}
\hline \hline $\begin{array}{c}\mathrm{d} \\
\mathrm{kpc}\end{array}$ & $\begin{array}{c}R_{\mathrm{GC}} \\
\mathrm{kpc}\end{array}$ & $\begin{array}{c}V_{\mathrm{LSR}} \\
\mathrm{km} \mathrm{s}^{-1}\end{array}$ & $\begin{array}{c}V_{\text {rad }} \\
\mathrm{km} \mathrm{s}^{-1}\end{array}$ \\
\hline 5.0 & 11.0 & 55 & 73 \\
7.5 & 13.0 & 77 & 96 \\
10.0 & 15.2 & 95 & 113 \\
12.5 & 17.4 & 108 & 126 \\
15.0 & 19.7 & 119 & 137 \\
17.5 & 22.0 & 127 & 146 \\
20.0 & 24.4 & 135 & 153 \\
\hline
\end{tabular}


Table 2. Photometry of field red giants in the direction of the galactic southern warp

\begin{tabular}{lcccccc}
\hline \hline Star ID & $\mathrm{x}$ & $\mathrm{y}$ & $V$ & $\sigma$ & $V-I_{C}$ & $\sigma$ \\
\hline 6194 & 632.35 & 549.11 & 12.069 & 0.001 & 0.852 & 0.001 \\
7245 & 743.09 & 639.93 & 12.238 & 0.001 & 0.654 & 0.001 \\
8235 & 568.60 & 723.38 & 12.263 & 0.001 & 0.839 & 0.001 \\
8414 & 821.97 & 739.55 & 12.294 & 0.001 & 0.618 & 0.001 \\
9037 & 970.74 & 791.66 & 12.518 & 0.001 & 0.643 & 0.001 \\
8621 & 909.56 & 758.48 & 12.570 & 0.001 & 0.524 & 0.001 \\
6326 & 729.49 & 559.45 & 12.583 & 0.001 & 0.852 & 0.001 \\
8622 & 954.65 & 757.60 & 12.647 & 0.001 & 0.641 & 0.001 \\
7013 & 955.57 & 617.85 & 12.698 & 0.001 & 0.717 & 0.001 \\
6403 & 704.46 & 566.64 & 12.725 & 0.001 & 0.804 & 0.001 \\
6266 & 945.53 & 554.63 & 12.820 & 0.001 & 1.224 & 0.001 \\
4897 & 933.43 & 435.79 & 12.901 & 0.002 & 0.574 & 0.003 \\
6380 & 781.51 & 564.95 & 12.913 & 0.002 & 1.065 & 0.002 \\
6688 & 874.70 & 590.07 & 12.915 & 0.002 & 1.699 & 0.002 \\
1178 & 977.60 & 110.65 & 12.928 & 0.002 & 0.683 & 0.003 \\
10289 & 605.47 & 899.63 & 12.934 & 0.002 & 1.119 & 0.002 \\
6216 & 847.26 & 550.49 & 12.986 & 0.002 & 0.590 & 0.003 \\
4021 & 698.82 & 362.46 & 13.033 & 0.002 & 0.657 & 0.003 \\
6062 & 933.35 & 536.45 & 13.045 & 0.002 & 0.937 & 0.003 \\
8766 & 594.12 & 771.19 & 13.112 & 0.002 & 0.577 & 0.003 \\
\hline
\end{tabular}

Note. - Table 2 is published in its entirety in the electronic edition of the Astrophysical Journal. A portion is shown here for guidance regarding its form and content. 
Table 3. Radial Velocities and Optical/IR Photometry for Stars Observed with the Argus Spectrograph

\begin{tabular}{|c|c|c|c|c|c|c|c|c|c|c|}
\hline Star ID & R. A. & DEC. & $V$ & $V-I_{C}$ & $K^{\mathrm{a}}$ & $J-K^{\mathrm{a}}$ & $H-K^{\mathrm{a}}$ & $V-K$ & $V_{\mathrm{rad}}$ & Notes \\
\hline 11015 & $7: 39: 35.1$ & $-31: 08: 31$ & 13.48 & 1.32 & 12.81 & 0.21 & 0.03 & 0.67 & 69.3 & 1 \\
\hline 9999 & $7: 39: 46.9$ & $-30: 59: 12$ & 14.00 & 1.33 & 10.86 & 0.70 & 0.14 & 3.14 & 112.7 & \\
\hline 5234 & $7: 40: 46.1$ & $-30: 43: 45$ & 14.06 & 1.49 & 10.40 & 0.89 & 0.18 & 3.66 & 93.7 & \\
\hline 4338 & $7: 40: 55.5$ & $-31: 08: 54$ & 14.06 & 1.43 & 10.64 & 0.81 & 0.13 & 3.42 & 85.5 & \\
\hline 5218 & $7: 40: 45.9$ & $-30: 51: 15$ & 14.06 & 1.30 & 10.92 & 0.72 & 0.10 & 3.14 & 96.2 & \\
\hline 7647 & $7: 40: 15.6$ & $-30: 55: 11$ & 14.06 & 1.25 & 11.15 & 0.68 & 0.11 & 2.91 & 70.8 & \\
\hline 6884 & $7: 40: 25.8$ & $-30: 44: 21$ & 14.10 & 1.41 & 10.74 & 0.80 & 0.15 & 3.36 & 68.4 & \\
\hline 7904 & $7: 40: 12.8$ & $-30: 51: 22$ & 14.10 & 1.32 & 10.91 & 0.74 & 0.15 & 3.19 & 67.9 & \\
\hline 4199 & $7: 40: 57.6$ & $-31: 01: 49$ & 14.16 & 1.01 & 11.83 & 0.56 & 0.08 & 2.33 & 17.6 & \\
\hline 9830 & $7: 39: 48.8$ & $-31: 01: 29$ & 14.22 & 1.32 & 10.99 & 0.80 & 0.21 & 3.23 & 62.9 & \\
\hline 7116 & $7: 40: 22.9$ & $-30: 40: 27$ & 14.25 & 1.32 & 11.13 & 0.71 & 0.13 & 3.12 & 73.8 & \\
\hline 7559 & $7: 40: 17.6$ & $-30: 40: 07$ & 14.28 & 1.45 & 10.81 & 0.84 & 0.18 & 3.47 & 114.3 & \\
\hline 879 & $7: 41: 40.2$ & $-30: 48: 32$ & 14.29 & 1.70 & 10.18 & 1.00 & 0.21 & 4.11 & 96.0 & \\
\hline 3917 & $7: 41: 00.9$ & $-31: 04: 01$ & 14.30 & 1.22 & 11.43 & 0.65 & 0.15 & 2.87 & 75.5 & \\
\hline 3373 & $7: 41: 07.5$ & $-31: 03: 41$ & 14.32 & 1.29 & 11.25 & 0.75 & 0.14 & 3.07 & 100.0 & \\
\hline 11027 & $7: 39: 35.2$ & $-30: 50: 29$ & 14.36 & 1.37 & 11.04 & 0.79 & 0.18 & 3.32 & 83.9 & \\
\hline 868 & $7: 41: 40.3$ & $-30: 48: 46$ & 14.38 & 1.42 & 11.02 & 0.77 & 0.22 & 3.36 & 107.9 & \\
\hline 9878 & $7: 39: 48.4$ & $-30: 55: 43$ & 14.39 & 1.64 & 10.38 & 1.03 & 0.22 & 4.01 & 64.6 & \\
\hline 5181 & $7: 40: 46.8$ & $-30: 40: 27$ & 14.40 & 1.27 & 11.35 & 0.73 & 0.15 & 3.05 & 93.7 & \\
\hline 6689 & $7: 40: 28.4$ & $-30: 41: 28$ & 14.42 & 1.47 & 10.90 & 0.83 & 0.16 & 3.52 & 76.6 & \\
\hline 1543 & $7: 41: 31.7$ & $-30: 48: 34$ & 14.45 & 1.70 & 10.36 & 1.00 & 0.19 & 4.09 & 112.7 & \\
\hline 8744 & $7: 40: 02.0$ & $-30: 54: 54$ & 14.46 & 1.35 & 11.12 & 0.80 & 0.18 & 3.34 & 100.8 & \\
\hline 3782 & $7: 41: 02.8$ & $-30: 56: 08$ & 14.47 & 1.18 & 11.62 & 0.68 & 0.14 & 2.85 & 87.5 & \\
\hline 5052 & $7: 40: 46.9$ & $-31: 08: 13$ & 14.49 & 1.59 & 10.71 & 0.91 & 0.20 & 3.78 & 85.5 & \\
\hline 4822 & $7: 40: 50.1$ & $-31: 01: 09$ & 14.50 & 1.39 & 11.16 & 0.82 & 0.16 & 3.34 & 43.3 & \\
\hline 2241 & $7: 41: 22.1$ & $-30: 54: 08$ & 14.50 & 1.27 & 11.55 & 0.67 & 0.15 & 2.95 & 96.8 & \\
\hline 11350 & $7: 39: 31.4$ & $-30: 53: 29$ & 14.51 & 1.61 & 10.54 & 1.00 & 0.16 & 3.97 & 78.8 & \\
\hline 2854 & $7: 41: 14.9$ & $-30: 47: 55$ & 14.52 & 1.42 & 11.15 & 0.80 & 0.12 & 3.37 & 101.3 & \\
\hline 10635 & $7: 39: 39.8$ & $-30: 51: 09$ & 14.52 & 1.42 & 11.03 & 0.90 & 0.16 & 3.49 & 75.7 & \\
\hline 6673 & $7: 40: 27.4$ & $-31: 01: 53$ & 14.53 & 1.36 & 11.23 & 0.83 & 0.19 & 3.30 & 40.5 & \\
\hline 9060 & $7: 39: 58.3$ & $-30: 57: 40$ & 14.54 & 1.23 & 11.60 & 0.65 & 0.11 & 2.94 & 130.4 & \\
\hline 8368 & $7: 40: 07.1$ & $-30: 48: 54$ & 14.56 & 1.34 & 11.41 & 0.67 & 0.14 & 3.15 & 59.4 & \\
\hline 10330 & $7: 39: 42.8$ & $-30: 56: 39$ & 14.59 & 1.29 & 11.46 & 0.75 & 0.19 & 3.13 & 93.0 & \\
\hline 7530 & $7: 40: 16.5$ & $-31: 05: 30$ & 14.59 & 1.27 & 11.54 & 0.74 & 0.16 & 3.05 & 75.7 & \\
\hline 263 & $7: 41: 47.7$ & $-30: 59: 42$ & 14.60 & 1.13 & 11.98 & 0.66 & 0.09 & 2.62 & 41.5 & \\
\hline 3697 & $7: 41: 04.8$ & $-30: 39: 24$ & 14.62 & 1.27 & 11.62 & 0.65 & 0.13 & 3.00 & 57.9 & \\
\hline 6554 & $7: 40: 28.7$ & $-31: 01: 12$ & 14.62 & 1.36 & 11.38 & 0.76 & 0.13 & 3.24 & 54.6 & \\
\hline
\end{tabular}


Table 3-Continued

\begin{tabular}{|c|c|c|c|c|c|c|c|c|c|c|}
\hline Star ID & R. A. & DEC. & $V$ & $V-I_{C}$ & $K^{\mathrm{a}}$ & $J-K^{\mathrm{a}}$ & $H-K^{\mathrm{a}}$ & $V-K$ & $V_{\text {rad }}$ & Notes \\
\hline 6755 & $7: 40: 27.5$ & $-30: 41: 44$ & 14.63 & 1.28 & 11.60 & 0.71 & 0.16 & 3.03 & 50.5 & \\
\hline 11102 & $7: 39: 34.7$ & $-30: 45: 47$ & 14.65 & 1.42 & 11.10 & 0.89 & 0.20 & 3.55 & 92.6 & \\
\hline 10808 & $7: 39: 37.5$ & $-30: 56: 02$ & 14.69 & 1.26 & 11.67 & 0.69 & 0.17 & 3.02 & 75.3 & \\
\hline 5153 & $7: 40: 46.5$ & $-30: 53: 32$ & 14.70 & 1.23 & 11.76 & 0.69 & 0.16 & 2.94 & 68.9 & \\
\hline 5814 & $7: 40: 37.5$ & $-31: 08: 04$ & 14.71 & 1.18 & 11.91 & 0.64 & 0.10 & 2.80 & 59.3 & \\
\hline 7333 & $7: 40: 20.2$ & $-30: 42: 11$ & 14.72 & 1.70 & 10.64 & 0.98 & 0.18 & 4.08 & 40.3 & \\
\hline 1336 & $7: 41: 34.4$ & $-30: 43: 40$ & 14.72 & 1.55 & 10.99 & 0.90 & 0.23 & 3.73 & 87.1 & \\
\hline 8216 & $7: 40: 09.7$ & $-30: 42: 28$ & 14.73 & 1.36 & 11.54 & 0.74 & 0.13 & 3.19 & 33.0 & \\
\hline 1164 & $7: 41: 35.4$ & $-31: 07: 28$ & 14.73 & 1.32 & 11.66 & 0.68 & 0.10 & 3.07 & 53.9 & \\
\hline 10044 & $7: 39: 46.3$ & $-30: 58: 37$ & 14.74 & 1.30 & 11.62 & 0.75 & 0.19 & 3.12 & 93.9 & \\
\hline 2831 & $7: 41: 15.2$ & $-30: 47: 20$ & 14.74 & 1.38 & 11.51 & 0.79 & 0.15 & 3.23 & 109.9 & \\
\hline 4735 & $7: 40: 51.0$ & $-31: 05: 11$ & 14.75 & 1.26 & 11.78 & 0.71 & 0.14 & 2.97 & 41.7 & \\
\hline 1465 & $7: 41: 31.7$ & $-31: 08: 40$ & 14.75 & 1.36 & 11.53 & 0.81 & 0.17 & 3.22 & 101.3 & \\
\hline 1507 & $7: 41: 32.0$ & $-30: 53: 09$ & 14.78 & 1.10 & 12.16 & 0.68 & 0.10 & 2.62 & 111.9 & \\
\hline 2547 & $7: 41: 17.6$ & $-31: 08: 08$ & 14.79 & 1.07 & 12.45 & 0.45 & 0.13 & 2.34 & 44.0 & \\
\hline 8516 & $7: 40: 04.8$ & $-30: 55: 10$ & 14.80 & 1.34 & 11.67 & 0.72 & 0.10 & 3.13 & 91.6 & \\
\hline 7073 & $7: 40: 23.1$ & $-30: 47: 50$ & 14.81 & 1.04 & 12.19 & 0.66 & 0.14 & 2.62 & 133.1 & \\
\hline 384 & $7: 41: 46.1$ & $-31: 03: 48$ & 14.83 & 1.41 & 11.53 & 0.77 & 0.17 & 3.30 & 95.8 & \\
\hline 5022 & $7: 40: 47.3$ & $-31: 08: 57$ & 14.84 & 1.36 & 11.60 & 0.77 & 0.14 & 3.23 & 97.0 & \\
\hline 7560 & $7: 40: 17.5$ & $-30: 39: 19$ & 14.84 & 1.81 & 10.55 & 1.03 & 0.18 & 4.29 & 67.2 & \\
\hline 1532 & $7: 41: 32.0$ & $-30: 41: 04$ & 14.85 & 1.37 & 11.65 & 0.69 & 0.12 & 3.20 & 73.4 & \\
\hline 8855 & $7: 40: 00.7$ & $-30: 56: 01$ & 14.85 & 1.35 & 11.77 & 0.71 & 0.15 & 3.08 & 88.0 & \\
\hline 10195 & $7: 39: 44.7$ & $-30: 52: 51$ & 14.85 & 1.34 & 11.60 & 0.80 & 0.20 & 3.25 & 94.9 & \\
\hline 8910 & $7: 39: 59.5$ & $-31: 05: 52$ & 14.85 & 1.21 & 12.00 & 0.74 & 0.18 & 2.85 & 42.9 & \\
\hline 3470 & $7: 41: 06.1$ & $-31: 05: 58$ & 14.91 & 1.14 & 12.19 & 0.70 & 0.12 & 2.72 & 60.2 & \\
\hline 6789 & $7: 40: 26.6$ & $-30: 53: 11$ & 14.91 & 1.16 & 12.22 & 0.62 & 0.09 & 2.69 & 55.4 & \\
\hline 10610 & $7: 39: 39.8$ & $-30: 55: 21$ & 14.91 & 1.32 & 11.69 & 0.76 & 0.16 & 3.22 & 93.6 & \\
\hline 5034 & $7: 40: 47.8$ & $-30: 54: 02$ & 14.92 & 1.38 & 11.59 & 0.82 & 0.11 & 3.33 & 75.3 & \\
\hline 8403 & $7: 40: 07.2$ & $-30: 40: 00$ & 14.92 & 1.46 & 11.52 & 0.78 & 0.16 & 3.40 & 76.4 & \\
\hline 9305 & $7: 39: 55.8$ & $-30: 47: 21$ & 14.93 & 1.02 & 12.63 & 0.48 & 0.09 & 2.30 & 27.5 & \\
\hline 2025 & $7: 41: 25.1$ & $-30: 53: 11$ & 14.93 & 1.29 & 11.90 & 0.68 & 0.08 & 3.03 & 94.5 & \\
\hline 446 & $7: 41: 46.0$ & $-30: 45: 57$ & 14.94 & 1.67 & 11.10 & 0.85 & 0.21 & 3.84 & 118.7 & \\
\hline 6756 & $7: 40: 27.7$ & $-30: 39: 57$ & 14.94 & 1.27 & 11.97 & 0.83 & 0.17 & 2.97 & 83.3 & \\
\hline 3234 & $7: 41: 10.0$ & $-30: 46: 34$ & 14.95 & 1.60 & 11.14 & 0.90 & 0.21 & 3.81 & 93.5 & \\
\hline 3278 & $7: 41: 09.4$ & $-30: 49: 18$ & 14.95 & 1.70 & 10.93 & 1.01 & 0.26 & 4.02 & 119.2 & \\
\hline 6447 & $7: 40: 29.7$ & $-31: 04: 54$ & 14.97 & 1.42 & 11.52 & 0.84 & 0.18 & 3.45 & 75.6 & \\
\hline 1045 & $7: 41: 37.5$ & $-31: 00: 41$ & 15.02 & 1.08 & 12.53 & 0.61 & 0.06 & 2.49 & 59.2 & \\
\hline
\end{tabular}


Table 3-Continued

\begin{tabular}{|c|c|c|c|c|c|c|c|c|c|c|}
\hline Star ID & R. A. & DEC. & $V$ & $V-I_{C}$ & $K^{\mathrm{a}}$ & $J-K^{\mathrm{a}}$ & $H-K^{\mathrm{a}}$ & $V-K$ & $V_{\text {rad }}$ & Notes \\
\hline 10148 & $7: 39: 45.9$ & $-31: 08: 48$ & 15.02 & 1.31 & 12.23 & 0.74 & 0.17 & 2.79 & 56.4 & \\
\hline 353 & $7: 41: 47.1$ & $-30: 51: 38$ & 15.02 & 1.42 & 11.73 & 0.78 & 0.16 & 3.29 & 44.0 & \\
\hline 283 & $7: 41: 48.0$ & $-30: 49: 21$ & 15.06 & 1.79 & 10.77 & 1.07 & 0.26 & 4.29 & 129.6 & \\
\hline 7512 & $7: 40: 17.9$ & $-30: 43: 45$ & 15.07 & 1.55 & 11.34 & 0.93 & 0.19 & 3.73 & 140.6 & \\
\hline 4884 & $7: 40: 49.9$ & $-30: 52: 30$ & 15.07 & 1.35 & 11.88 & 0.71 & 0.09 & 3.19 & 114.7 & \\
\hline 8181 & $7: 40: 10.0$ & $-30: 44: 13$ & 15.07 & 1.37 & 12.10 & 0.71 & 0.17 & 2.97 & 63.7 & \\
\hline 7632 & $7: 40: 16.2$ & $-30: 46: 13$ & 15.10 & 1.10 & 12.80 & 0.50 & 0.17 & 2.30 & 60.6 & \\
\hline 806 & $7: 41: 40.5$ & $-31: 00: 35$ & 15.10 & 1.64 & 11.26 & 0.89 & 0.19 & 3.84 & 104.2 & \\
\hline 1261 & $7: 41: 34.5$ & $-30: 59: 16$ & 15.10 & 1.11 & 12.59 & 0.57 & 0.04 & 2.51 & 17.3 & \\
\hline 2541 & $7: 41: 18.5$ & $-30: 51: 46$ & 15.11 & 1.37 & 11.83 & 0.79 & 0.15 & 3.28 & 76.6 & \\
\hline 329 & $7: 41: 47.5$ & $-30: 46: 50$ & 15.14 & 1.61 & 11.33 & 0.92 & 0.22 & 3.81 & 121.5 & \\
\hline 1962 & $7: 41: 25.3$ & $-31: 04: 38$ & 15.14 & 1.31 & 12.09 & 0.69 & 0.14 & 3.05 & 102.6 & \\
\hline 6474 & $7: 40: 30.2$ & $-30: 48: 49$ & 15.14 & 1.00 & 12.94 & 0.42 & 0.04 & 2.20 & 38.1 & \\
\hline 2954 & $7: 41: 13.9$ & $-30: 43: 58$ & 15.14 & 1.54 & 11.45 & 0.85 & 0.17 & 3.69 & 108.4 & \\
\hline 9186 & $7: 39: 56.8$ & $-30: 55: 47$ & 15.16 & 1.04 & 13.03 & 0.44 & 0.05 & 2.13 & 46.7 & \\
\hline 2490 & $7: 41: 18.9$ & $-30: 58: 35$ & 15.19 & 1.64 & 11.67 & 0.80 & 0.18 & 3.52 & 19.7 & \\
\hline 10138 & $7: 39: 44.9$ & $-31: 00: 38$ & 15.19 & 1.63 & 11.19 & 0.98 & 0.21 & 4.00 & 127.2 & \\
\hline 529 & $7: 41: 45.0$ & $-30: 40: 51$ & 15.21 & 1.41 & 11.87 & 0.79 & 0.22 & 3.34 & 101.5 & \\
\hline 10906 & $7: 39: 35.8$ & $-31: 03: 33$ & 15.21 & 1.21 & 12.32 & 0.64 & 0.17 & 2.89 & 57.4 & \\
\hline 5658 & $7: 40: 41.0$ & $-30: 44: 20$ & 15.23 & 1.26 & 12.19 & 0.73 & 0.14 & 3.04 & 99.8 & \\
\hline 7342 & $7: 40: 19.8$ & $-30: 46: 03$ & 15.23 & 1.27 & 12.36 & 0.69 & 0.13 & 2.87 & 61.1 & \\
\hline 7420 & $7: 40: 18.9$ & $-30: 48: 40$ & 15.25 & 1.10 & 12.52 & 0.67 & 0.19 & 2.73 & 98.8 & \\
\hline 9820 & $7: 39: 49.6$ & $-30: 49: 56$ & 15.25 & 1.17 & 12.55 & 0.60 & 0.10 & 2.70 & 72.0 & \\
\hline 3713 & $7: 41: 03.6$ & $-31: 00: 35$ & 15.27 & 1.16 & 12.60 & 0.63 & 0.15 & 2.67 & 42.4 & \\
\hline 11233 & $7: 39: 33.1$ & $-30: 49: 04$ & 15.29 & 1.80 & 13.55 & 0.52 & 0.07 & 1.74 & 32.4 & 2 \\
\hline 6189 & $7: 40: 33.2$ & $-31: 01: 07$ & 15.29 & 1.10 & 12.73 & 0.62 & 0.06 & 2.56 & 63.6 & \\
\hline 4166 & $7: 40: 58.3$ & $-30: 54: 09$ & 15.30 & 1.46 & 11.79 & 0.89 & 0.14 & 3.51 & 153.4 & \\
\hline 2223 & $7: 41: 22.1$ & $-30: 59: 31$ & 15.30 & 1.03 & 13.11 & 0.40 & 0.16 & 2.19 & 51.3 & \\
\hline 5296 & $7: 40: 45.6$ & $-30: 41: 13$ & 15.32 & 1.27 & 12.27 & 0.79 & 0.13 & 3.05 & 66.2 & \\
\hline 2213 & $7: 41: 22.0$ & $-31: 04: 09$ & 15.33 & 1.52 & 11.78 & 0.82 & 0.14 & 3.55 & 43.7 & \\
\hline 10815 & $7: 39: 37.6$ & $-30: 40: 07$ & 15.33 & 1.64 & 11.35 & 1.04 & 0.20 & 3.98 & 99.8 & \\
\hline 4326 & $7: 40: 57.1$ & $-30: 41: 35$ & 15.35 & 1.33 & 12.15 & 0.74 & 0.16 & 3.20 & 100.7 & \\
\hline 2958 & $7: 41: 12.9$ & $-31: 04: 05$ & 15.35 & 1.40 & 12.06 & 0.78 & 0.16 & 3.29 & 92.6 & \\
\hline 4009 & $7: 41: 00.7$ & $-30: 49: 18$ & 15.35 & 1.08 & 12.90 & 0.53 & 0.10 & 2.45 & 47.4 & \\
\hline 6282 & $7: 40: 31.8$ & $-31: 04: 59$ & 15.36 & 1.41 & 11.99 & 0.82 & 0.16 & 3.37 & 95.8 & \\
\hline 9954 & $7: 39: 48.4$ & $-30: 41: 49$ & 15.37 & 1.04 & 13.08 & 0.46 & 0.08 & 2.29 & 39.9 & \\
\hline 1214 & $7: 41: 35.2$ & $-31: 00: 07$ & 15.37 & 1.43 & 12.13 & 0.76 & 0.15 & 3.24 & 128.4 & \\
\hline
\end{tabular}


Table 3-Continued

\begin{tabular}{|c|c|c|c|c|c|c|c|c|c|c|}
\hline Star ID & R. A. & DEC. & $V$ & $V-I_{C}$ & $K^{\mathrm{a}}$ & $J-K^{\mathrm{a}}$ & $H-K^{\mathrm{a}}$ & $V-K$ & $V_{\text {rad }}$ & Notes \\
\hline 9392 & $7: 39: 55.0$ & $-30: 43: 21$ & 15.37 & 1.33 & 12.19 & 0.79 & 0.12 & 3.18 & 106.5 & \\
\hline 1759 & $7: 41: 28.9$ & $-30: 47: 13$ & 15.37 & 1.53 & 11.73 & 0.89 & 0.27 & 3.64 & 137.3 & \\
\hline 462 & $7: 41: 45.8$ & $-30: 43: 42$ & 15.38 & 1.56 & 11.73 & 0.86 & 0.21 & 3.65 & 129.2 & \\
\hline 2679 & $7: 41: 16.4$ & $-31: 03: 33$ & 15.39 & 1.38 & 12.18 & 0.70 & 0.14 & 3.21 & 121.3 & \\
\hline 2809 & $7: 41: 15.2$ & $-30: 56: 14$ & 15.40 & 1.39 & 12.18 & 0.74 & 0.15 & 3.22 & 93.5 & \\
\hline 7010 & $7: 40: 23.3$ & $-31: 01: 02$ & 15.42 & 1.34 & 12.18 & 0.76 & 0.14 & 3.24 & 101.8 & \\
\hline 8989 & $7: 39: 58.9$ & $-31: 03: 41$ & 15.42 & 1.04 & 12.81 & 0.60 & 0.45 & 2.61 & 24.6 & 3 \\
\hline 916 & $7: 41: 40.0$ & $-30: 40: 31$ & 15.43 & 1.25 & 12.59 & 0.67 & 0.12 & 2.84 & 25.4 & \\
\hline 6737 & $7: 40: 27.5$ & $-30: 44: 45$ & 15.44 & 1.21 & 12.84 & 0.61 & 0.06 & 2.60 & 55.3 & \\
\hline 5220 & $7: 40: 46.1$ & $-30: 48: 12$ & 15.44 & 1.46 & 11.97 & 0.89 & 0.21 & 3.47 & 37.5 & \\
\hline 7249 & $7: 40: 19.6$ & $-31: 08: 47$ & 15.44 & 1.21 & 12.66 & 0.62 & 0.07 & 2.78 & 57.2 & \\
\hline 2507 & $7: 41: 19.3$ & $-30: 44: 16$ & 15.45 & 1.56 & 11.75 & 0.89 & 0.22 & 3.70 & 107.3 & \\
\hline 9987 & $7: 39: 46.7$ & $-31: 05: 27$ & 15.46 & 1.34 & 12.26 & 0.77 & 0.17 & 3.20 & 85.6 & \\
\hline 10026 & $7: 39: 45.9$ & $-31: 08: 48$ & 15.46 & 1.37 & 12.23 & 0.74 & 0.17 & 3.23 & 110.9 & \\
\hline 10205 & $7: 39: 44.2$ & $-31: 01: 27$ & 15.47 & 1.36 & 12.22 & 0.75 & 0.17 & 3.25 & 107.1 & \\
\hline 9004 & $7: 39: 58.8$ & $-31: 01: 02$ & 15.47 & 1.22 & 12.57 & 0.72 & 0.12 & 2.90 & 71.4 & \\
\hline 6725 & $7: 40: 27.8$ & $-30: 42: 10$ & 15.48 & 1.80 & 11.16 & 1.06 & 0.21 & 4.32 & 112.2 & \\
\hline 4058 & $7: 41: 00.2$ & $-30: 44: 35$ & 15.49 & 1.35 & 12.27 & 0.77 & 0.11 & 3.22 & 83.8 & \\
\hline 100 & $7: 41: 50.8$ & $-30: 41: 21$ & 15.49 & 1.49 & 12.03 & 0.79 & 0.18 & 3.46 & 63.2 & \\
\hline 6643 & $7: 40: 28.8$ & $-30: 43: 43$ & 15.50 & 1.90 & 12.39 & 0.81 & 0.16 & 3.11 & 67.2 & 4 \\
\hline 8257 & $7: 40: 08.8$ & $-30: 47: 15$ & 15.52 & 1.31 & 12.37 & 0.82 & 0.15 & 3.15 & 99.3 & 5 \\
\hline 9917 & $7: 39: 48.7$ & $-30: 45: 01$ & 15.53 & 1.43 & 12.14 & 0.80 & 0.12 & 3.39 & 41.6 & \\
\hline 10923 & $7: 39: 37.1$ & $-30: 40: 53$ & 15.54 & 1.51 & 12.05 & 0.82 & 0.16 & 3.49 & 72.8 & \\
\hline 10877 & $7: 39: 37.6$ & $-30: 40: 07$ & 15.58 & 1.77 & 11.35 & 1.04 & 0.20 & 4.23 & 120.2 & \\
\hline 3897 & $7: 41: 01.3$ & $-31: 02: 03$ & 15.59 & 1.14 & 12.98 & 0.54 & 0.12 & 2.61 & 37.1 & \\
\hline 3242 & $7: 41: 09.7$ & $-30: 50: 45$ & 15.60 & 1.05 & 13.13 & 0.59 & 0.10 & 2.47 & 33.5 & \\
\hline 6019 & $7: 40: 35.5$ & $-30: 59: 16$ & 15.60 & 1.47 & 12.15 & 0.77 & 0.15 & 3.45 & 65.0 & \\
\hline 4523 & $7: 40: 54.5$ & $-30: 42: 19$ & 15.61 & 1.28 & 12.59 & 0.70 & 0.12 & 3.02 & 116.5 & \\
\hline 4167 & $7: 40: 58.6$ & $-30: 46: 51$ & 15.64 & 1.28 & 12.55 & 0.73 & 0.13 & 3.09 & 87.6 & \\
\hline 2617 & $7: 41: 17.6$ & $-30: 52: 00$ & 15.70 & 1.70 & 11.71 & 0.98 & 0.21 & 3.99 & 109.7 & \\
\hline 2121 & $7: 41: 23.5$ & $-30: 58: 14$ & 15.72 & 1.46 & 12.34 & 0.75 & 0.12 & 3.38 & 105.4 & \\
\hline 767 & $7: 41: 41.5$ & $-30: 48: 06$ & 15.73 & 1.02 & 13.45 & 0.48 & 0.12 & 2.28 & 56.4 & \\
\hline 1295 & $7: 41: 35.0$ & $-30: 41: 18$ & 15.73 & 1.03 & 13.43 & 0.45 & 0.10 & 2.30 & 33.9 & \\
\hline 11084 & $7: 39: 34.7$ & $-30: 46: 39$ & 15.74 & 1.39 & 12.38 & 0.79 & 0.18 & 3.36 & 101.3 & \\
\hline 5386 & $7: 40: 44.4$ & $-30: 42: 24$ & 15.74 & 1.54 & 12.06 & 0.85 & 0.13 & 3.68 & 137.0 & \\
\hline 5967 & $7: 40: 35.4$ & $-31: 00: 26$ & 15.74 & 1.18 & 11.74 & 0.36 & 0.07 & 4.00 & 49.7 & 6 \\
\hline 5631 & $7: 40: 41.2$ & $-30: 46: 25$ & 15.74 & 1.11 & 13.44 & 0.60 & 0.05 & 2.30 & 76.8 & \\
\hline
\end{tabular}


Table 3-Continued

\begin{tabular}{|c|c|c|c|c|c|c|c|c|c|c|}
\hline Star ID & R. A. & DEC. & $V$ & $V-I_{C}$ & $K^{\mathrm{a}}$ & $J-K^{\mathrm{a}}$ & $H-K^{\mathrm{a}}$ & $V-K$ & $V_{\text {rad }}$ & Notes \\
\hline 2055 & $7: 41: 24.1$ & $-31: 05: 25$ & 15.75 & 1.96 & 11.07 & 1.14 & 0.26 & 4.68 & 118.8 & \\
\hline 8280 & $7: 40: 07.7$ & $-30: 59: 49$ & 15.76 & 1.28 & 12.68 & 0.71 & 0.10 & 3.08 & 122.2 & \\
\hline 2199 & $7: 41: 23.3$ & $-30: 41: 00$ & 15.77 & 1.55 & 12.09 & 0.86 & 0.21 & 3.68 & 78.0 & \\
\hline 3956 & 7:41:01.4 & $-30: 43: 24$ & 15.78 & 1.47 & 12.22 & 0.89 & 0.15 & 3.56 & 93.1 & \\
\hline 8070 & $7: 40: 11.4$ & $-30: 39: 32$ & 15.79 & 1.07 & 13.55 & 0.44 & 0.07 & 2.24 & 51.1 & \\
\hline 8978 & $7: 39: 59.4$ & $-30: 55: 13$ & 15.81 & 1.28 & 12.80 & 0.69 & 0.09 & 3.01 & 65.4 & \\
\hline 724 & $7: 41: 42.2$ & $-30: 43: 37$ & 15.81 & 1.62 & 11.98 & 0.95 & 0.17 & 3.84 & 75.3 & \\
\hline 10787 & $7: 39: 38.4$ & $-30: 45: 46$ & 15.82 & 1.44 & 12.41 & 0.77 & 0.10 & 3.41 & 102.7 & \\
\hline 9577 & $7: 39: 52.4$ & $-30: 48: 53$ & 15.84 & 1.41 & 12.50 & 0.80 & 0.18 & 3.34 & 59.4 & \\
\hline 8631 & $7: 40: 04.1$ & $-30: 44: 14$ & 15.86 & 1.37 & 12.64 & 0.73 & 0.08 & 3.22 & 116.7 & \\
\hline 6950 & $7: 40: 23.8$ & $-31: 01: 32$ & 15.86 & 1.40 & 12.49 & 0.85 & 0.17 & 3.37 & 124.5 & \\
\hline 7235 & $7: 40: 19.9$ & $-31: 04: 31$ & 15.95 & 1.38 & 12.66 & 0.78 & 0.12 & 3.29 & 64.9 & \\
\hline 8874 & $7: 39: 60.0$ & $-31: 04: 40$ & 15.95 & 1.36 & 12.66 & 0.79 & 0.18 & 3.29 & 85.2 & \\
\hline 5472 & $7: 40: 43.2$ & $-30: 45: 40$ & 15.98 & 1.42 & 12.57 & 0.82 & 0.14 & 3.41 & 102.5 & \\
\hline 2927 & $7: 41: 13.9$ & $-30: 51: 29$ & 16.00 & 1.28 & 13.05 & 0.66 & 0.13 & 2.95 & 50.7 & \\
\hline 6587 & $7: 40: 28.1$ & $-31: 05: 11$ & 16.02 & 1.57 & 12.32 & 0.86 & 0.18 & 3.70 & 142.5 & \\
\hline 294 & $7: 41: 48.0$ & $-30: 43: 40$ & 16.04 & 1.21 & 13.20 & 0.72 & 0.17 & 2.84 & 24.1 & \\
\hline 1084 & $7: 41: 37.1$ & $-30: 56: 36$ & 16.04 & 1.29 & 13.29 & 0.77 & 0.14 & 2.75 & 91.6 & \\
\hline 10055 & $7: 39: 46.6$ & $-30: 51: 42$ & 16.10 & 1.24 & 13.31 & 0.65 & 0.11 & 2.79 & 49.5 & \\
\hline 11459 & $7: 39: 29.9$ & $-30: 56: 18$ & 16.11 & 1.35 & 13.25 & 0.74 & 0.18 & 2.86 & 55.3 & \\
\hline 5196 & $7: 40: 46.7$ & $-30: 39: 35$ & 16.11 & 1.34 & 12.96 & 0.72 & 0.11 & 3.15 & 102.8 & \\
\hline 9405 & $7: 39: 54.8$ & $-30: 45: 34$ & 16.12 & 1.62 & 12.33 & 0.90 & 0.09 & 3.79 & 97.8 & \\
\hline 396 & $7: 41: 45.9$ & $-31: 05: 37$ & 16.13 & 1.25 & 13.34 & 0.63 & 0.13 & 2.79 & 45.2 & \\
\hline 9912 & $7: 39: 47.8$ & $-30: 59: 31$ & 16.13 & 1.07 & 13.69 & 0.55 & 0.14 & 2.44 & 33.5 & \\
\hline 9978 & $7: 39: 47.2$ & $-30: 56: 56$ & 16.13 & 1.25 & 13.16 & 0.76 & 0.26 & 2.97 & 16.6 & \\
\hline 11436 & $7: 39: 30.9$ & $-30: 45: 06$ & 16.13 & 1.10 & 13.83 & 0.61 & 0.23 & 2.30 & 74.7 & \\
\hline 6486 & $7: 40: 30.3$ & $-30: 43: 53$ & 16.15 & 1.11 & 13.87 & 0.52 & 0.13 & 2.28 & 57.5 & \\
\hline 1179 & $7: 41: 35.3$ & $-31: 05: 56$ & 16.15 & 1.13 & 14.48 & 0.44 & 0.03 & 1.67 & 72.1 & 7 \\
\hline 8569 & $7: 40: 04.8$ & $-30: 43: 27$ & 16.16 & 1.33 & 13.09 & 0.68 & 0.12 & 3.07 & 93.6 & \\
\hline 11107 & $7: 39: 33.5$ & $-31: 03: 51$ & 16.19 & 1.44 & 12.71 & 0.85 & 0.20 & 3.48 & 125.8 & \\
\hline 1258 & $7: 41: 35.4$ & $-30: 39: 22$ & 16.19 & 1.36 & 12.95 & 0.73 & 0.13 & 3.24 & 99.6 & \\
\hline 7413 & $7: 40: 18.1$ & $-31: 02: 55$ & 16.22 & 1.29 & 13.23 & 0.73 & 0.13 & 2.99 & 56.5 & \\
\hline 1148 & $7: 41: 36.0$ & $-31: 01: 16$ & 16.23 & 1.01 & 13.72 & 0.87 & 0.13 & 2.51 & 56.8 & 8 \\
\hline 7387 & $7: 40: 19.6$ & $-30: 42: 09$ & 16.24 & 1.43 & 13.28 & 0.78 & 0.16 & 2.96 & 29.9 & \\
\hline 6645 & $7: 40: 28.9$ & $-30: 40: 30$ & 16.28 & 1.02 & 13.99 & 0.53 & 0.03 & 2.29 & 74.0 & \\
\hline 8136 & 7:40:09.2 & $-31: 04: 04$ & 16.29 & 1.15 & 13.47 & 0.69 & 0.17 & 2.82 & 84.6 & \\
\hline 3744 & $7: 41: 03.6$ & $-30: 51: 27$ & 16.29 & 1.05 & 13.90 & 0.48 & 0.15 & 2.39 & 103.8 & \\
\hline
\end{tabular}


Table 3 - Continued

\begin{tabular}{lccccccccccc}
\hline \hline Star ID & R. A. & DEC. & $V$ & $V-I_{C}$ & $K^{\text {a }}$ & $J-K^{\text {a }}$ & $H-K^{\text {a }}$ & $V-K$ & $V_{\text {rad }}$ & Notes \\
\hline 4730 & $7: 40: 52.0$ & $-30: 48: 16$ & 16.29 & 1.32 & 13.11 & 0.75 & 0.15 & 3.18 & 82.3 & \\
5665 & $7: 40: 39.8$ & $-31: 04: 43$ & 16.30 & 1.35 & 13.10 & 0.83 & 0.22 & 3.20 & 125.3 & 9 \\
9124 & $7: 39: 57.2$ & $-31: 01: 24$ & 16.31 & 1.39 & 12.92 & 0.84 & 0.19 & 3.39 & 126.4 & \\
6704 & $7: 40: 27.0$ & $-31: 00: 18$ & 16.32 & 1.42 & 12.87 & 0.83 & 0.18 & 3.45 & 91.9 & \\
2285 & $7: 41: 21.2$ & $-30: 59: 48$ & 16.32 & 1.48 & 12.92 & 0.76 & 0.17 & 3.40 & 103.1 & \\
1436 & $7: 41: 32.7$ & $-30: 53: 52$ & 16.33 & 1.03 & 14.11 & 0.40 & 0.00 & 2.22 & 70.2 & \\
1636 & $7: 41: 30.2$ & $-30: 57: 20$ & 16.36 & 1.38 & 13.09 & 0.78 & 0.17 & 3.27 & 110.6 & \\
2727 & $7: 41: 16.1$ & $-30: 56: 36$ & 16.36 & 1.04 & 14.16 & 0.49 & 0.05 & 2.20 & 105.1 & \\
6232 & $7: 40: 32.4$ & $-31: 04: 12$ & 16.37 & 1.38 & 13.03 & 0.76 & 0.06 & 3.34 & 112.3 & \\
8641 & $7: 40: 03.2$ & $-30: 56: 57$ & 16.37 & 1.27 & 13.37 & 0.67 & 0.10 & 3.00 & 79.1 & \\
8887 & $7: 39: 59.7$ & $-31: 06: 14$ & 16.38 & 1.35 & 13.23 & 0.74 & 0.18 & 3.15 & 39.8 & \\
558 & $7: 41: 43.8$ & $-31: 02: 12$ & 16.39 & 1.55 & 12.76 & 0.78 & 0.14 & 3.63 & 58.3 & \\
2864 & $7: 41: 14.5$ & $-30: 53: 06$ & 16.40 & 1.29 & 13.32 & 0.71 & 0.15 & 3.08 & 116.3 & \\
11563 & $7: 39: 28.9$ & $-30: 51: 45$ & 16.40 & 1.33 & 13.36 & 0.68 & 0.11 & 3.04 & 76.6 & \\
11399 & $7: 39: 31.1$ & $-30: 49: 41$ & 16.41 & 1.09 & 14.02 & 0.66 & 0.12 & 2.39 & 39.2 & \\
2969 & $7: 41: 12.8$ & $-31: 02: 22$ & 16.42 & 1.45 & 13.07 & 0.74 & 0.16 & 3.35 & 127.9 & \\
8346 & $7: 40: 07.6$ & $-30: 45: 02$ & 16.44 & 1.62 & 13.10 & 0.74 & 0.15 & 3.34 & 42.0 & \\
7600 & $7: 40: 16.9$ & $-30: 42: 34$ & 16.45 & 1.07 & 14.02 & 0.55 & 0.15 & 2.43 & 19.5 & \\
3004 & $7: 41: 12.7$ & $-30: 54: 42$ & 16.45 & 1.52 & 13.24 & 0.71 & 0.04 & 3.21 & 43.8 & \\
2717 & $7: 41: 16.6$ & $-30: 47: 15$ & 16.46 & 1.45 & 13.15 & 0.89 & 0.21 & 3.31 & 66.9 & \\
9200 & $7: 39: 56.2$ & $-31: 02: 27$ & 16.47 & 1.33 & 13.21 & 0.77 & 0.15 & 3.26 & 79.5 & \\
955 & $7: 41: 39.1$ & $-30: 48: 16$ & 16.47 & 1.00 & 14.13 & 0.56 & 0.25 & 2.34 & 44.8 & \\
3751 & $7: 41: 03.6$ & $-31: 00: 35$ & 16.47 & 1.48 & 12.60 & 0.63 & 0.15 & 3.87 & 102.2 & \\
5547 & $7: 40: 42.5$ & $-30: 41: 25$ & 16.50 & 1.03 & 14.09 & 0.72 & 0.21 & 2.41 & 38.1 & \\
\hline
\end{tabular}

${ }^{\text {a}}$ From 2MASS

Note. - 1. Uncertain photometry \& identification. 2. Uncertain photometry. 3. Uncertain 2MASS photometry. 4 . $K=7.3 \mathrm{mag}$ star $8^{\prime \prime}$ away. 5 . $K$ magnitude is uncertain. 6 . $K=6.5$ mag star $13^{\prime \prime}$ away. 7. Uncertain photometry \& identification. 8. Uncertain photometry \& identification: $K=14.4$ mag star $4^{\prime \prime}$ away. 9. Uncertain photometry: $K=14.2 \mathrm{mag} 4^{\prime \prime}$ away. 
Table 4. Field Giants in the Southern Warp: Spectroscopic data

\begin{tabular}{cccccccc}
\hline \hline Star & $\ell$ & $b$ & $\begin{array}{c}\text { HJD } \\
-2400000\end{array}$ & $\begin{array}{c}\text { Exp. Time } \\
(\mathrm{min})\end{array}$ & $\mathrm{S} / \mathrm{N}$ & $\begin{array}{c}V_{\mathrm{rad}} \\
\left(\mathrm{km} \mathrm{s}^{-1}\right)\end{array}$ & $\begin{array}{c}V_{\mathrm{GSR}} \\
\left(\mathrm{km} \mathrm{s}^{-1}\right)\end{array}$ \\
\hline \multirow{2}{*}{9060} & 245.71 & -4.25 & 51203.84616 & $2 \times 60$ & 59 & $+132.9 \pm 1.5$ & -86.0 \\
& & & 51204.85843 & $2 \times 60$ & 49 & & \\
7512 & 245.55 & -4.08 & 50834.65961 & $5 \times 60$ & 77 & $+139.8 \pm 0.5$ & -78.8 \\
4166 & 245.77 & -4.04 & 50833.68964 & $5 \times 60$ & 71 & $+156.2 \pm 0.5$ & -62.7 \\
\hline
\end{tabular}


Table 5. Atmospheric Parameters

\begin{tabular}{rrrrrrrr}
\hline \hline Star & {$[\mathrm{Fe} / \mathrm{H}]^{\mathrm{a}}$} & $T_{\text {eff }}{ }^{\mathrm{b}}$ & $\log \mathrm{g}^{\mathrm{b}}$ & $T_{\text {eff }}{ }^{\mathrm{c}}$ & $\log \mathrm{g}^{\mathrm{c}}$ & $V_{\text {turb }}$ & {$[\mathrm{Fe} / \mathrm{H}]^{\mathrm{c}}$} \\
\hline 9060 & -0.6 & 5480 & 1.4 & 5250 & 3.0 & 1.5 & -0.48 \\
7512 & -0.6 & 4750 & 1.0 & 4590 & 2.25 & 1.4 & -0.40 \\
4166 & -0.6 & 4860 & 1.0 & 4590 & 2.2 & 1.6 & -0.42 \\
\hline
\end{tabular}

${ }^{a}$ Adopted value based on the Galactic metallicity gradient.

${ }^{b}$ Estimates obtained using the reddening estimate from Schlegel et al. (1998) and distance estimates based on the radial velocity and an adopted circular Galactic rotation.

${ }^{\mathrm{c}}$ Quantities derived using the spectroscopic methods described in the text. 
Table 6. Stellar Atomic Line Data

\begin{tabular}{|c|c|c|c|c|c|c|c|c|c|c|c|c|c|c|}
\hline$\lambda(\AA)$ & Species & $\operatorname{LEP}(\mathrm{eV})$ & $\log g f$ & Source & $\lambda(\AA)$ & Species & $\operatorname{LEP}(\mathrm{eV})$ & $\log g f$ & Source & $\lambda(\AA)$ & Species & $\operatorname{LEP}(\mathrm{eV})$ & $\log g f$ & Source \\
\hline 6300.30 & 8.0 & 0.00 & -9.717 & AG04 & 5855.09 & 26.0 & 4.60 & -1.547 & PS03 & 6575.02 & 26.0 & 2.59 & -2.727 & OXF \\
\hline 6363.78 & 8.0 & 0.02 & -10.185 & AG04 & 5856.10 & 26.0 & 4.29 & -1.640 & PSO3 & 6581.21 & 26.0 & 1.50 & -4.680 & ANOO \\
\hline 5688.19 & 11.0 & 2.11 & -0.420 & $\mathrm{RC} 02$ & 5858.79 & 26.0 & 4.22 & -2.260 & PSO3 & 6609.11 & 26.0 & 2.56 & -2.692 & OXF \\
\hline 6154.23 & 11.0 & 2.10 & -1.530 & $\mathrm{RC} 02$ & 5909.97 & 26.0 & 3.21 & -2.640 & OXF & 6648.08 & 26.0 & 1.01 & -5.918 & OXF \\
\hline 6160.75 & 11.0 & 2.10 & -1.230 & $\mathrm{RC} 02$ & 5916.25 & 26.0 & 2.45 & -2.994 & OXF & 6699.16 & 26.0 & 4.59 & -2.170 & OXF \\
\hline 6318.72 & 12.0 & 5.11 & -1.970 & $\mathrm{RC} 02$ & 5927.80 & 26.0 & 4.65 & -1.090 & PS03 & 6739.52 & 26.0 & 1.56 & -4.820 & OXF \\
\hline 6319.24 & 12.0 & 5.11 & -2.220 & $\mathrm{RC} 02$ & 5933.80 & 26.0 & 4.64 & $\begin{array}{l}-2.230 \\
-2.030\end{array}$ & PSO3 & 6750.15 & 26.0 & 2.42 & $\begin{array}{l}-2.621 \\
\end{array}$ & OXF \\
\hline 6965.41 & 12.0 & 5.75 & -1.510 & KB95 & 5956.69 & 26.0 & 0.86 & -4.608 & OXF & 6786.86 & 26.0 & 4.19 & -1.850 & TF00 \\
\hline 7387.69 & 12.0 & 5.75 & -0.870 & $\mathrm{RC} 02$ & 6012.21 & 26.0 & 2.22 & -4.070 & OXF & 6810.26 & 26.0 & 4.60 & -1.000 & OXF \\
\hline 6696.02 & 13.0 & 3.14 & -1.340 & $\mathrm{RC} 02$ & 6019.36 & 26.0 & 3.57 & -3.360 & PSO3 & 6971.93 & 26.0 & 3.02 & -3.390 & OXF \\
\hline 6698.67 & 13.0 & 3.14 & -1.640 & $\mathrm{RC} 02$ & 6027.05 & 26.0 & 4.07 & -1.106 & OXF & 7112.17 & 26.0 & $\begin{array}{l}.02 \\
2.99\end{array}$ & $\begin{array}{l}-0.090 \\
-3.044\end{array}$ & OXF \\
\hline 7835.31 & 13.0 & 4.02 & -0.470 & LUCK & 6054.08 & 26.0 & 4.37 & -2.310 & PSO3 & 7223.66 & 26.0 & 3.01 & -2.269 & OXF \\
\hline 7836.13 & 13.0 & 4.02 & -0.310 & $\mathrm{RC} 02$ & 6105.13 & 26.0 & 4.55 & -2.050 & PSO3 & 5991.38 & 26.1 & 3.15 & -3.557 & BB91 \\
\hline 5690.43 & 14.0 & 4.93 & -1.751 & SOLAR & 6120.24 & 26.0 & 0.91 & -5.970 & OXF & 6084.11 & 26.1 & 3.20 & -3.808 & BB91 \\
\hline 5793.07 & 14.0 & 4.93 & -1.843 & SOLAR & 6145.42 & 26.0 & 3.37 & -3.600 & TF00 & 6149.26 & 26.1 & 3.89 & -2.724 & BB91 \\
\hline 6125.02 & 14.0 & 5.61 & -1.506 & SOLAR & 6151.62 & 26.0 & 2.17 & -3.299 & OXF & 6247.56 & 26.1 & 3.89 & -2.329 & BB91 \\
\hline 6145.01 & 14.0 & 5.62 & -1.362 & SOLAR & 6157.73 & 26.0 & 4.08 & -1.320 & TF00 & 6369.46 & 26.1 & 2.89 & -4.250 & BB91 \\
\hline 6155.13 & 14.0 & 5.62 & -0.786 & SOLAR & 6159.38 & 26.0 & 4.61 & -1.970 & PSO3 & 6416.92 & 26.1 & 3.89 & -2.740 & BB91 \\
\hline 6166.44 & 20.0 & 2.52 & -1.142 & OXF & 6165.36 & 26.0 & 4.14 & -1.490 & OXF & 6432.68 & 26.1 & 2.89 & -3.708 & BB91 \\
\hline 6169.04 & 20.0 & 2.52 & -0.797 & OXF & 6173.34 & 26.0 & 2.22 & -2.880 & OXF & 6456.38 & 26.1 & 3.90 & -2.075 & BB91 \\
\hline 6169.56 & 20.0 & 2.53 & -0.478 & OXF & 6180.20 & 26.0 & 2.73 & -2.637 & OXF & 6189.00 & 27.0 & 1.71 & -2.450 & PNOO \\
\hline 6455.60 & 20.0 & 2.52 & -1.290 & OXF & 6200.31 & 26.0 & 2.61 & -2.437 & OXF & 6455.03 & 27.0 & 3.63 & -0.250 & PNOO \\
\hline 6064.63 & 22.0 & 1.05 & -1.888 & OXF & 6219.28 & 26.0 & 2.20 & -2.433 & OXF & 6632.45 & 27.0 & 2.28 & -2.000 & PNOO \\
\hline 6091.17 & 22.0 & 2.27 & -0.367 & OXF & 6229.23 & 26.0 & 2.84 & -2.846 & OXF & 5846.99 & 28.0 & 1.68 & -3.210 & $\mathrm{RC} 02$ \\
\hline 6312.24 & 22.0 & 1.46 & -1.496 & OXF & 6232.64 & 26.0 & $\begin{array}{l}3.04 \\
3.65\end{array}$ & $\begin{array}{l}-1.040 \\
-1.283\end{array}$ & OXF & 6086.28 & 28.0 & $\begin{array}{l}1.00 \\
4.26\end{array}$ & -0.515 & $\mathrm{RC} 02$ \\
\hline 6336.10 & 22.0 & 1.44 & -1.687 & OXF & 6246.32 & 26.0 & 3.60 & -0.894 & OXF & 6175.37 & 28.0 & 4.09 & -0.535 & $\mathrm{RC} 02$ \\
\hline 6013.53 & 25.0 & 3.07 & -0.250 & PM00 & 6265.13 & 26.0 & 2.17 & -2.550 & OXF & 6177.24 & 28.0 & 1.83 & -3.510 & $\mathrm{RC} 02$ \\
\hline 6016.67 & 25.0 & 3.08 & -0.220 & PMO0 & 6270.22 & 26.0 & 2.86 & -2.500 & OXF & 6204.60 & 28.0 & 4.09 & -1.140 & $\mathrm{RC} 02$ \\
\hline 6021.80 & 25.0 & 3.08 & 0.030 & PMO0 & 6271.28 & 26.0 & 3.33 & -2.703 & ANOO & 6635.12 & 28.0 & 4.42 & -0.828 & $\mathrm{RC} 02$ \\
\hline 5618.63 & 26.0 & 4.21 & -1.292 & OXF & 6297.79 & 26.0 & 2.22 & -2.740 & OXF & 6772.32 & 28.0 & 3.66 & -0.987 & $\mathrm{RC} 02$ \\
\hline 5701.55 & 26.0 & 2.56 & -2.216 & OXF & 6301.50 & 26.0 & 3.65 & -0.766 & OXF & 7800.00 & 37.0 & 0.00 & 0.130 & TL99 \\
\hline 5705.47 & 26.0 & 4.30 & -1.420 & OXF & 6322.69 & 26.0 & 2.59 & -2.426 & OXF & 6127.44 & 40.0 & 0.15 & -1.060 & $\mathrm{RC} 02$ \\
\hline 5741.85 & 26.0 & 4.25 & -1.689 & OXF & 6336.82 & 26.0 & 3.68 & -0.916 & OXF & 6134.55 & 40.0 & 0.00 & -1.280 & $\mathrm{RC} 02$ \\
\hline 5775.08 & 26.0 & 4.22 & -1.310 & OXF & 6353.84 & 26.0 & 0.91 & -6.477 & OXF & 6143.20 & 40.0 & 0.07 & -1.100 & $\mathrm{RC} 02$ \\
\hline 5778.45 & 26.0 & 2.59 & -3.480 & OXF & 6355.03 & 26.0 & 2.84 & -2.403 & OXF & 5853.64 & 56.1 & 0.60 & -1.010 & PNOO \\
\hline 5811.92 & 26.0 & 4.14 & -2.430 & PS03 & 6411.65 & 26.0 & 3.65 & -0.734 & OXF & 5805.77 & 57.1 & 0.13 & -1.560 & LB01 \\
\hline 5837.70 & 26.0 & 4.29 & -2.340 & TF00 & 6469.19 & 26.0 & 4.84 & -0.770 & TF00 & 6390.48 & 57.1 & 0.32 & -1.410 & LB01 \\
\hline 5853.16 & 26.0 & 1.49 & -5.280 & PS03 & 6574.23 & 26.0 & 0.99 & -5.004 & OXF & 6645.11 & 63.1 & 1.38 & 0.120 & LW01 \\
\hline
\end{tabular}

References. - AG04 = Asplund et al. ( 2004); AN00 = Asplund et al. (2000); BB91 = Biemont et al. (1991); KB05 = Kurucz \& Bell (1995); LB01 = Lawler, Bonvallet, \& Sneden (2000); LW01 = Lawler et al. (2001); LUCK = Luck (private communication); OXF = Group at Oxford (Smith \& Raggett 1981, Blackwell et al. 1979a,b; 1980; 1982a,b;

SOLAR = Inverted Solar analysis; TF00 = Thorén \& Feltzing (2000); TL99 = Tomkin \& Lambert (1999). 
Table 7. Mean stellar abundances

\begin{tabular}{|c|c|c|c|c|c|c|c|c|c|}
\hline Species & Abundance & $\sigma$ & $\mathrm{N}$ & Abundance & $\sigma$ & $\mathrm{N}$ & Abundance & $\sigma$ & $\mathrm{N}$ \\
\hline & \multicolumn{3}{|c|}{4166} & \multicolumn{3}{|c|}{7521} & \multicolumn{3}{|c|}{9060} \\
\hline$[\mathrm{O} / \mathrm{Fe}]$ & 0.19 & 0.14 & 2 & 0.15 & 0.11 & 2 & 0.44 & 0.07 & 2 \\
\hline$[\mathrm{Na} / \mathrm{Fe}]$ & 0.24 & 0.08 & 3 & 0.28 & 0.18 & 3 & 0.37 & 0.10 & 3 \\
\hline$[\mathrm{Mg} / \mathrm{Fe}]$ & 0.34 & 0.06 & 4 & 0.24 & 0.04 & 4 & 0.21 & 0.06 & 4 \\
\hline$[\mathrm{Al} / \mathrm{Fe}]$ & 0.30 & 0.04 & 2 & 0.26 & 0.10 & 4 & 0.15 & 0.03 & 4 \\
\hline$[\mathrm{Si} / \mathrm{Fe}]$ & 0.19 & 0.07 & 5 & 0.18 & 0.08 & 5 & 0.12 & 0.11 & 5 \\
\hline$[\mathrm{Ca} / \mathrm{Fe}]$ & 0.25 & 0.16 & 4 & 0.29 & 0.14 & 4 & 0.03 & 0.03 & 4 \\
\hline$[\mathrm{Ti} / \mathrm{Fe}]$ & 0.23 & 0.07 & 4 & 0.33 & 0.10 & 4 & 0.41 & 0.06 & 3 \\
\hline$[\mathrm{Mn} / \mathrm{Fe}]$ & -0.29 & 0.10 & 3 & -0.23 & 0.11 & 3 & -0.23 & 0.11 & 3 \\
\hline$[\mathrm{FeI} / \mathrm{H}]$ & -0.45 & 0.17 & 38 & -0.43 & 0.20 & 41 & -0.51 & 0.14 & 44 \\
\hline$[\mathrm{FeII} / \mathrm{H}]$ & -0.49 & 0.07 & 8 & -0.47 & 0.04 & 7 & -0.53 & 0.14 & 8 \\
\hline$[\mathrm{Co} / \mathrm{Fe}]$ & 0.10 & 0.18 & 3 & 0.16 & 0.09 & 3 & 0.26 & 0.19 & 3 \\
\hline$[\mathrm{Ni} / \mathrm{Fe}]$ & 0.06 & 0.13 & 7 & 0.07 & 0.11 & 7 & 0.03 & 0.11 & 7 \\
\hline$[\mathrm{Rb} / \mathrm{Fe}]$ & 0.10 & $\ldots$ & 1 & 0.14 & $\ldots$ & 1 & $\ldots$ & $\ldots$ & $\ldots$ \\
\hline$[\mathrm{Zr} / \mathrm{Fe}]$ & -0.12 & 0.04 & 3 & -0.08 & 0.17 & 3 & $\ldots$ & $\ldots$ & $\cdots$ \\
\hline$[\mathrm{Ba} / \mathrm{Fe}]$ & 0.16 & $\ldots$ & 1 & -0.03 & $\ldots$ & 1 & -0.14 & $\ldots$ & 1 \\
\hline$[\mathrm{La} / \mathrm{Fe}]$ & 0.36 & 0.01 & 2 & 0.27 & 0.04 & 2 & 0.26 & 0.07 & 2 \\
\hline$[\mathrm{Eu} / \mathrm{Fe}]$ & 0.46 & $\ldots$ & 1 & 0.39 & $\ldots$ & 1 & 0.66 & $\ldots$ & 1 \\
\hline
\end{tabular}


Table 8. Revised Distance Estimates

\begin{tabular}{rrrrrrrrr}
\hline \hline Star & $V-K$ & $(V-K)_{0}{ }^{\mathrm{a}}$ & $T_{\mathrm{eff}}^{\mathrm{a}}$ & $\mathrm{E}(B-V)$ & $M_{K}^{\mathrm{b}}$ & $(m-M)_{0}$ & $\mathrm{~d}^{\mathrm{c}}$ & $R_{\mathrm{GC}}{ }^{\mathrm{c}}$ \\
\hline 9060 & 2.94 & 1.90 & 5216 & 0.39 & -1.6 & 13.08 & 4.13 & 10.40 \\
7512 & 3.73 & 2.50 & 4581 & 0.45 & -3.2 & 14.39 & 7.55 & 13.07 \\
4166 & 3.51 & 2.50 & 4582 & 0.37 & -3.2 & 14.86 & 9.38 & 14.61 \\
\hline
\end{tabular}

a The de-reddened $V-K$ value is that needed to produce the $T_{\text {eff }}$ value cited here, and which agrees well with the value derived spectroscopically: see Table 5 .

${ }^{\mathrm{b}}$ The $M_{\mathrm{K}}$ value has been derived using the relation between this value and $T_{\text {eff }}$ for the open cluster Be 29, which has a very similar metallicity as the field stars, as described in the text.

${ }^{\mathrm{c}}$ Distances are given in kpc. 
Table 9. Abundance Comparisons ${ }^{\mathrm{a}}$

\begin{tabular}{|c|c|c|c|c|c|c|}
\hline Element & M67 means & $\sigma$ & Outer disk clusters ${ }^{\mathrm{b}}$ & $\sigma$ & Warp stars & $\sigma$ \\
\hline $\mathrm{O}$ & $+0.08 \pm 0.01$ & 0.03 & $+0.21 \pm 0.02$ & 0.04 & $+0.26 \pm 0.09$ & 0.16 \\
\hline $\mathrm{Na}$ & $+0.30 \pm 0.04$ & 0.07 & $+0.31 \pm 0.05$ & 0.11 & $+0.30 \pm 0.04$ & 0.07 \\
\hline $\mathrm{Mg}$ & $+0.16 \pm 0.01$ & 0.02 & $+0.30 \pm 0.03$ & 0.07 & $+0.26 \pm 0.04$ & 0.07 \\
\hline $\mathrm{Al}$ & $+0.17 \pm 0.01$ & 0.01 & $+0.21 \pm 0.02$ & 0.04 & $+0.24 \pm 0.05$ & 0.08 \\
\hline $\mathrm{Mg}$ & $+0.16 \pm 0.01$ & 0.02 & $+0.30 \pm 0.03$ & 0.07 & $+0.26 \pm 0.04$ & 0.07 \\
\hline $\mathrm{Si}$ & $+0.09 \pm 0.01$ & 0.02 & $+0.12 \pm 0.03$ & 0.08 & $+0.16 \pm 0.02$ & 0.04 \\
\hline $\mathrm{Ca}$ & $+0.07 \pm 0.02$ & 0.03 & $+0.07 \pm 0.02$ & 0.05 & $+0.19 \pm 0.08$ & 0.14 \\
\hline $\mathrm{Ti}$ & $+0.12 \pm 0.04$ & 0.06 & $+0.29 \pm 0.05$ & 0.13 & $+0.36 \pm 0.05$ & 0.09 \\
\hline $\mathrm{Mn}$ & $-0.13 \pm 0.04$ & 0.06 & $-0.24 \pm 0.07$ & 0.16 & $-0.25 \pm 0.02$ & 0.03 \\
\hline Co & $+0.02 \pm 0.01$ & 0.01 & $+0.14 \pm 0.03$ & 0.08 & $+0.17 \pm 0.05$ & 0.08 \\
\hline $\mathrm{Ni}$ & $+0.08 \pm 0.02$ & 0.03 & $+0.01 \pm 0.02$ & 0.06 & $+0.05 \pm 0.01$ & 0.02 \\
\hline \multirow[t]{3}{*}{$\mathrm{Rb}$} & $-0.27 \pm 0.03$ & 0.05 & $-0.09 \pm 0.05$ & 0.12 & $+0.12 \pm 0.02$ & 0.03 \\
\hline & & & $-0.04 \pm 0.08$ & 0.14 & & \\
\hline & & & $-0.16 \pm 0.05$ & 0.06 & & \\
\hline \multirow[t]{3}{*}{$\mathrm{Zr}$} & $-0.28 \pm 0.02$ & 0.03 & $+0.20 \pm 0.10$ & 0.24 & $-0.10 \pm 0.02$ & 0.03 \\
\hline & & & $+0.06 \pm 0.01$ & 0.03 & & \\
\hline & & & $+0.49 \pm 0.14$ & 0.20 & & \\
\hline \multirow[t]{3}{*}{$\mathrm{Ba}$} & $-0.02 \pm 0.02$ & 0.04 & $+0.41 \pm 0.13$ & 0.31 & $+0.00 \pm 0.09$ & 0.15 \\
\hline & & & $+0.22 \pm 0.05$ & 0.10 & & \\
\hline & & & $+0.78 \pm 0.14$ & 0.19 & & \\
\hline \multirow[t]{3}{*}{$\mathrm{La}$} & $+0.11 \pm 0.01$ & 0.02 & $+0.42 \pm 0.11$ & 0.27 & $+0.30 \pm 0.03$ & 0.06 \\
\hline & & & $+0.27 \pm 0.04$ & 0.07 & & \\
\hline & & & $+0.74 \pm 0.17$ & 0.24 & & \\
\hline \multirow[t]{3}{*}{$\mathrm{Eu}$} & $+0.06 \pm 0.02$ & 0.03 & $+0.29 \pm 0.06$ & 0.16 & $+0.50 \pm 0.08$ & 0.14 \\
\hline & & & $+0.23 \pm 0.05$ & 0.10 & & \\
\hline & & & $+0.37 \pm 0.20$ & 0.28 & & \\
\hline
\end{tabular}

${ }^{a}$ The values in parentheses are standard errors, while the quoted uncertainty is the error of the mean.

${ }^{\mathrm{b}}$ For the $s$-process elements $\mathrm{Rb}, \mathrm{Zr}, \mathrm{Ba}$, and $\mathrm{La}$, and the $r$-process element $\mathrm{Eu}$ we provide three means for the clusters. The first row is for all the stars in all the clusters. The second row includes results only for Be 20 and Be 29. The third row is for the two clusters with enhanced neutron capture elements, Be 31 and NGC 2141. 


\section{REFERENCES}

Alonso, A., Arribas, S., \& Martinez Roger, C. 1994, A\&AS, 107, 365

Alonso, A., Arribas, S., \& Martinez Roger, C. 1999, A\&AS, 140, 261

Alves, D. R. 2000, ApJ, 539, 732

Andrievsky, S. M., Bersier, D., Kovtyukh, V. V., Luck, R. E., Maciel, W. J., Lépine, J. R. D., \& Beletsky, Yu. V. 2002a, A\&A, 384, 140

Andrievsky, S. M., Kovtyukh, V. V., Luck, R. E., Lépine, J. R. D., Bersier, D., Maciel, W. J., Barbuy, B., Klochkova, V. G., Panchuk, V. E., \& Karpischek, R. U. 2002b, A\&A, 381,32

Andrievsky, S. M., Kovtyukh, V. V., Luck, R. E., Lépine, J. R. D., Maciel, W. J., \& Beletsky, Yu. V. 2002c, A\&A, 392, 491

Andrievsky, S. M., Luck, R. E., Martin, P., \& Lépine, J. R. D. 2003, A\&A, 413, 159

Asplund, M., Grevesse, N., Sauval, A. J., Allende Prieto, C., \& Kiselman, D. 2004, A\&A, 417,751

Asplund, M., Nordlund, Å., Trampedach, R., \& Stein, R. F. 2000, A\&A, 359, 743

Bensby, T., Feltzing, S., \& Lundström, I. 2003, A\&A, 410, 527

Bensby, T., Feltzing, S., \& Lundström, I. 2004, A\&A, 421, 969

Biémont, E., Baudoux, M., Kurucz, R. L., Ansbacher, W., \& Pinnington, E. H. 1991, A\&A, 249,539

Blackwell, D. E., Booth, A. J., Haddock, D. J., Petford, A. D., \& Legget, S. K. 1986a, MNRAS, 220, 549

Blackwell, D. E., Booth, A. J., Menon, S. L. R., \& Petford, A. D., 1986b, MNRAS, 220, 289

Blackwell, D. E., Ibbetson, P. A., Petford, A. D., \& Shallis, M. J. 1979a, MNRAS, 186, 633

Blackwell, D. E., Lynas-Gray, A. E., \& Smith, G. 1995, A\&A, 296, 217

Blackwell, D. E., Menon, S. L. R., \& Petford, A. D. 1983, MNRAS, 204, 883

Blackwell, D. E., Menon, S. L. R., Petford, A. D., \& Shallis, M. J. 1982a, MNRAS, 201, 611

Blackwell, D. E., Petford, A. D., \& Shallis, M. J. 1979b, MNRAS, 186, 657

Blackwell, D. E., Petford, A. D., Shallis, M. J., \& Legget, S. 1982b, MNRAS, 199, 21

Blackwell, D. E., Petford, A. D., Shallis, M. J., \& Simmons, G. J. 1980, MNRAS, 191, 445

Blair, W. P., Kirshner, R. P., \& Chevalier, R. A. 1982, ApJ, 254, 50

Brewer, M.-M., \& Carney, B. W. 2004, PASA, 21, 134 
Brewer, M.-M., \& Carney, B. W. 2005, AJ, submitted

Burton, W. B., \& Te Lintel Hekkert, P. 1986, A\&A, 65, 427

Carney, B. W., \& Seitzer, P. 1993, AJ, 105, 2127

Carpenter, J. M. 2001, AJ, 121, 2851

Chen, L. Hou, J. L., \& Wang, J. J. 2003, AJ, 125, 1397

Crane, J. D., Majewski, S. R., Rocha-Pinto, H. J., Frinchaboy, P. M., Skrutskie, M. F., \& Law, D. R. 2003, ApJ, 594, L119

Daflon, S., \& Cunha, K. 2004, ApJ, 617, 1115

Dennefeld, M., \& Kunth, D. 1981, AJ, 86, 989

Diplas, A., \& Savage, B. D. 1991, ApJ, 377, 126

Djorgovski, S., \& Sosin, C. 1989, ApJ, 341, L13

Fich, M., \& Silkey, M. 1991, ApJ, 366, 107

Freudenreich, H. T., Beriiman, G. B., Dwek, I., Hauser, M. G., Kelsall, T., Moseley, S. H., Silverberg, R. F., Sodroski, T. J., Toller, G. N., \& Weiland, J. L. 1994, ApJ, 429, L69

Frinchaboy, P. M., Majewski, S. R., Crane, J. D., Reid, I. N., Rocha-Pinto, H. J., Phelps, R. L., Patterson, R. J., \& Muñoz, R. R. 2004, ApJ, 602, L21

Fuhrmann, K. 1998, A\&A, 338, 161

Graham, J. A. 1982, PASP, 94, 244

Grevesse, N., \& Sauval, A. J. 1998, Space Sci. Reviews, 85, 161

Henderson, A. P., Jackson, P. D., \& Kerr, F. J. 1982, ApJ, 263, 116

Henry, R. B. C., \& Worthey, G. 1999, PASP, 111, 919

Ibata, R. A., Irwin, M. J., Lewis, G. F., Ferguson, A. M. N., \& Tanvir, N. 2003, MNRAS, 340, L21

Jacoby, G. H., \& Ciardullo, R. 1999, ApJ, 515, 169

Janes, K. A. 1979, ApJS, 39, 135

Kurucz, R. L., \& Bell, B. 1995, Kurucz CD-ROM No. 23: Smithsonian Astrophysical Observatory

Lambert, D. L., \& Luck, R. E. 1976, The Observatory, 96, 100

Lawler, J. E., Bonvallet, G., \& Sneden, C. 2001a, ApJ, 556, 452

Lawler, J. E., Wickliffe, M. E., den Hartog, E. A., \& Sneden, C. 2001b, ApJ, 563, 1075 
López-Corredoira, M., Cabrera-Lavers, A., Garzón, F., \& Hammersley, P. L. 2002, A\&A, 394,883

Luck, R. E., Gieren, W. P., Andrievsky, S. M., Kovtyukh, V. V., Fouqué, P., Pont, F., \& Kienzle, F. 2003, A\&A, 401, 939

Martin, N. F., Ibata, R. A., Bellazzini, M., Irwin, M. J., Lewis, G. F., \& Dehnen, W. 2004, MNRAS, 348, 12

Miyamoto, M., Yoshizawa, M., \& Suzuki, S. 1988, A\&A, 194, 107

Newberg, H. J., Yanny, B., Rockosi, C., Grebel, E. K., Rix, H.-H., Brinkmann, J., Csabai, I., Hennessy, G., Hindsley, R. B., Ibata, R., Ivezić, Z., Lamb, D., Nash, E. T., Odenkirchen, M., Rave, H. A., Schneider, D. P., Smyth, J. A., Stolte, A., \& York, D. G. 2002, ApJ, 569, 245

Orsatti, A. M. 1992, AJ, 104, 590

Pagel, B. E. J., \& Edmunds, M. G. 1981, ARA\&A, 19, 77

Paulson, D. B., Sneden, C., \& Cochran, W. D. 2003, AJ, 125, 318

Peñarrubia, J., Martínez-Delgado, D., Rix, H. W., Gómez-Flechoso, M. A., Munn, J., Newberg, H., Bell, E. F., Yanny, B., Zucker, D., \& Grebel, E. K. 2004, astro-ph/0410448

Prochaska, J. X., \& McWilliam, A. 2000, ApJ, 537, L57

Prochaska, J. X., Naumov, S. O., Carney, B. W., McWilliam, A., \& Wolfe, A. M. 2000, AJ, 120,2513

Ramírez, S. V., \& Cohen, J. G. 2002, AJ, 123, 3277

Reed, B. C. 1996, AJ, 111, 804

Rocha-Pinto, H. J., Majewski, S. R., Skrutskie, M. F., \& Crane, J. D. 2003, ApJ, 594, L115

Rolleston, W. R. J., Smartt, S. J., Dufton, P. L., \& Ryans, R. S. I. 2000, A\&A, 363, 537

Schlegel, D. J., Finkbeiner, D. P., \& Davis, M. 1998, ApJ, 500, 525

Shaver, P., McGee, R. X., Newton, L. M., Danks, A. C., \& Pottasch, S. R. 1983, MNRAS, 204, 53

Smith, G., \& Raggett, D. St. J. 1981, J. Phys. B, 14, 4015

Sneden, C. 1973, ApJ, 184, 839

Sodroski, T. J., Dwek, E., Hauser, M. G., \& Kerr, F. J. 1987, ApJ, 322, 101

Thorén, P., \& Feltzing, S. 2000, A\&A, 393, 692

Tomkin, J., \& Lambert, D. L. 1999, ApJ, 523, 234 
Trundle, C., Dufton, P. L., Lennon, D. J., Smartt, S. J., \& Urbaneja, M. A. 2002, A\&A, 395,519

Twarog, B. A., Ashman, K. M., \& Anthony-Twarog, B. J. 1997, AJ, 114, 2556

Venn, K. A., Irwin, M., Shetrone, M. D., Tout, C. A., Hill, V., \& Tolstoy, E. 2004, AJ, 128, 1177

Venn, K. A., McCarthy, J. K.,. Lennon, D. J., Przybilla, N., Kudritzki, R. P., \& Lemke, M. 2000, ApJ, 541, 510

Vílchez, J. M., \& Esteban, C. 1996, MNRAS, 280, 720

Wouterloot, J. G. A., Brand, J., Burton, W. B., \& Kwee, K. K. 1990, A\&A, 230, 21

Yanny, B., Newberg, H. J., Grebel, E. K., Kent, R., Odenkirchen, M., Rockosi, C. M., Schlegel, D., Subbarao, M., Brinkmann, J., Fukugita, M., Ivezić, Z., Lamb, D., Schneider, D. P., \& York, D. G. 2003, ApJ, 588, 824

Yong, D., Carney, B. W., \& de Almeida, M. L. T. 2005, AJ, submitted

Zaritsky, D., Kennicutt, Jr., R. C., \& Huchra, J. P. 1994, ApJ, 420, 87 


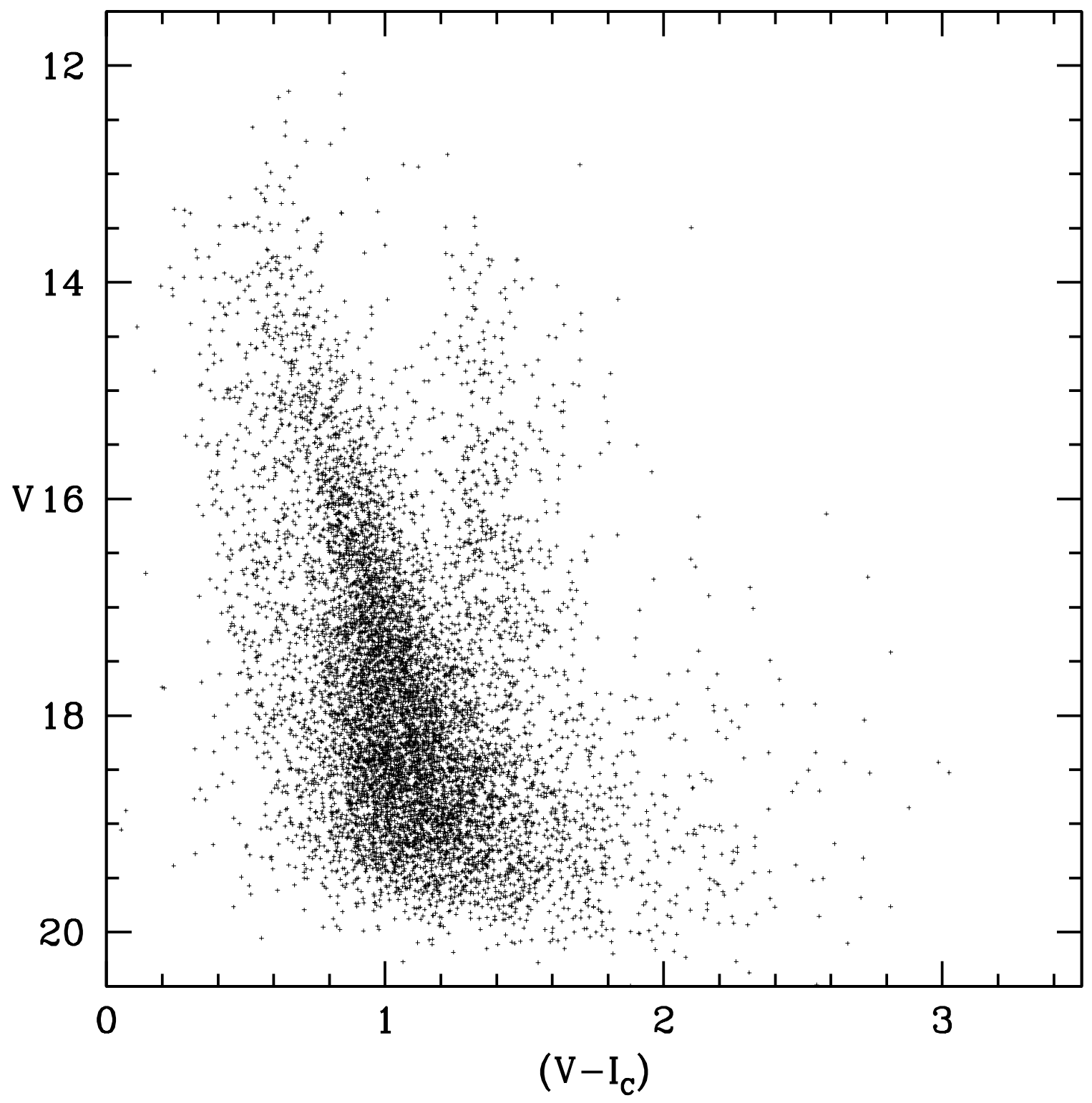

Fig. 1. - The color-magnitude diagram in the direction of Warp Field 1a (following Carney \& Seitzer (1993), covering a field of view of roughly 31 arcminutes. 
Fig. 2.- A view of Warp Field 1a, with expanded views of the regions surrounding our three primary targets. 


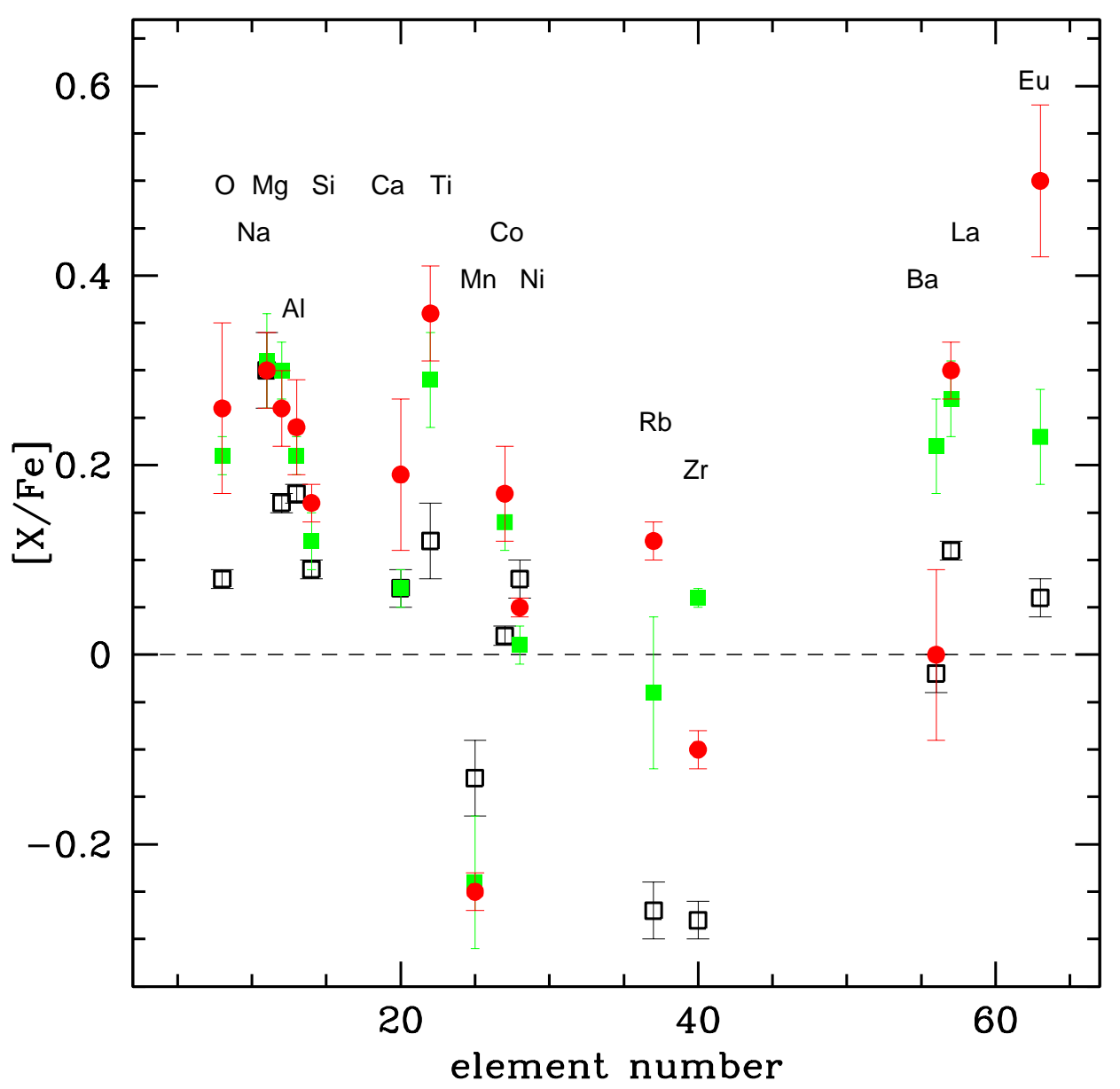

Fig. 3.- A comparison of the mean abundances for stars from Paper I and this paper. Plus signs represent the three stars in the old open cluster M67, filled green squares are the six stars in the old open clusters Be 20, Be 29, Be 31, and NGC 2141 in the outer Galactic disk, and the filled red circles are the field stars analyzed in this paper. For the elements Rb, Zr, $\mathrm{Ba}$, La, and Eu we show results only for Be 20 and Be 29, for reasons discussed in the text. 


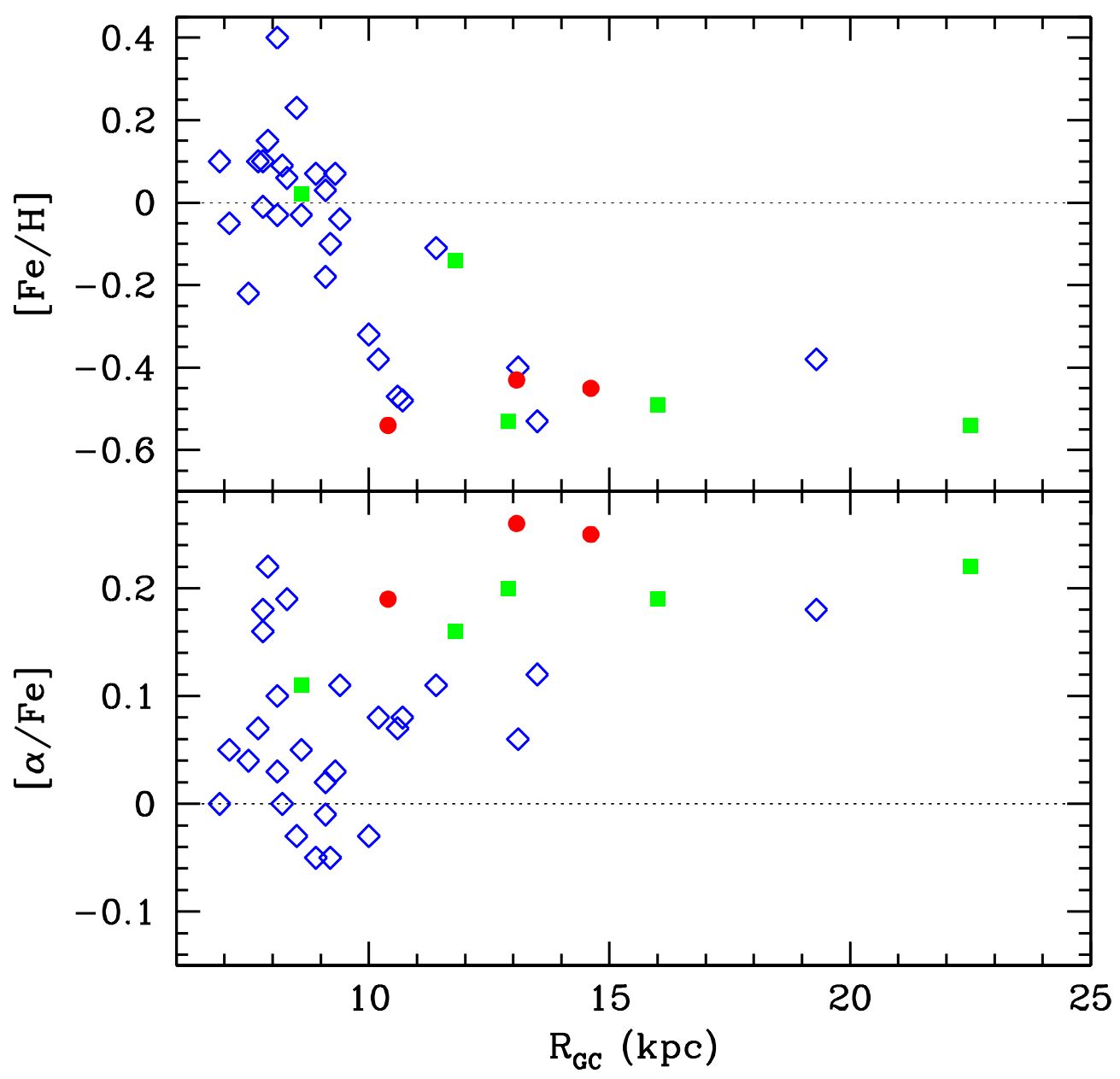

Fig. 4.- Upper: The metallicity gradient of old open clusters from the literature (blue diamonds), from Yong et al. (2005; green squares), and the three field stars discussed in this paper (filled red circles). Lower: The mean abundances of $\mathrm{Mg}, \mathrm{Si}, \mathrm{Ca}$, and $\mathrm{Ti}$ compared to iron, using the same symbols, as a function of Galactocentric distance. 


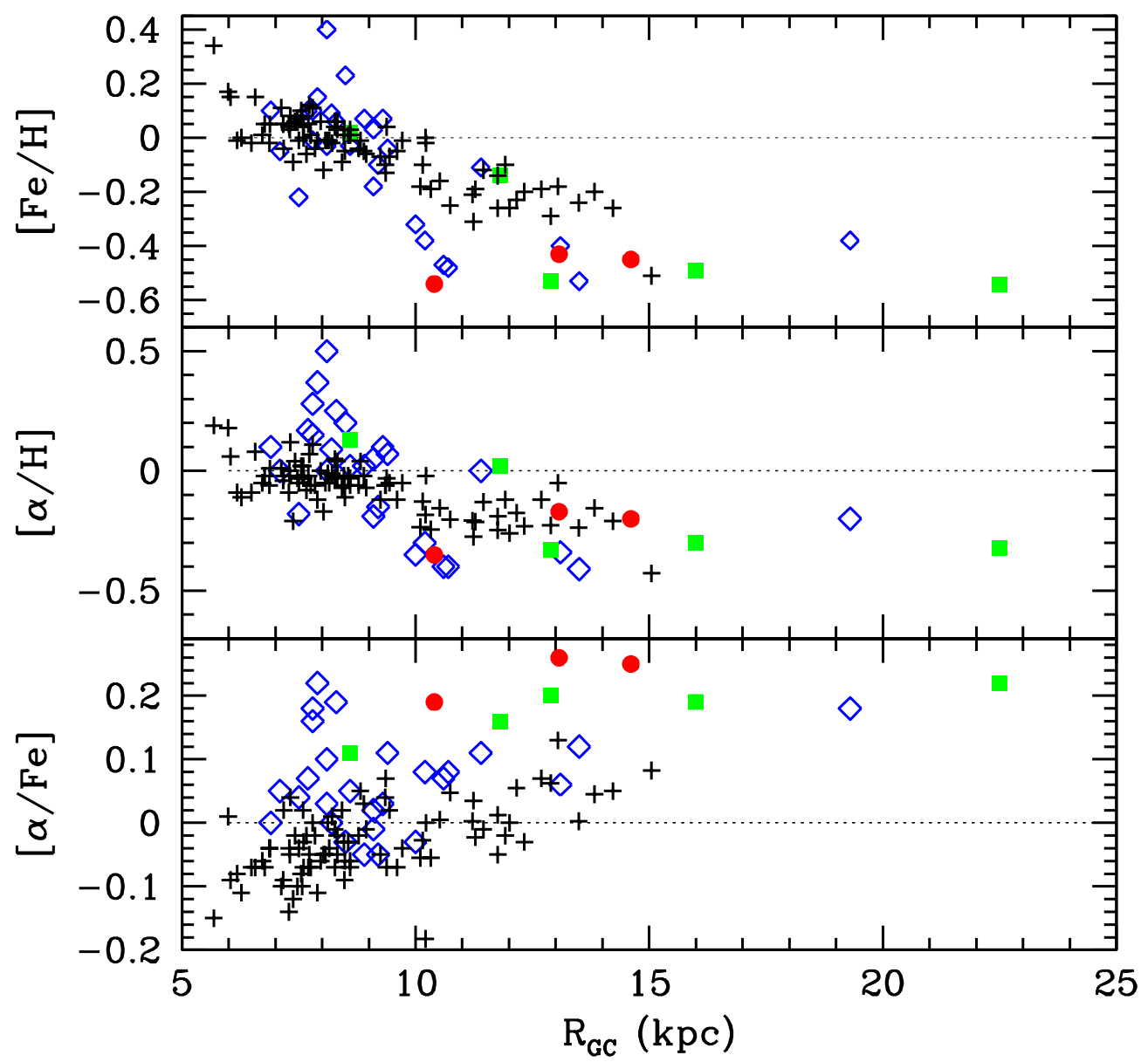

Fig. 5.- The same as Figure 4, but also including the results from Cepheid variables (black plus signs). 


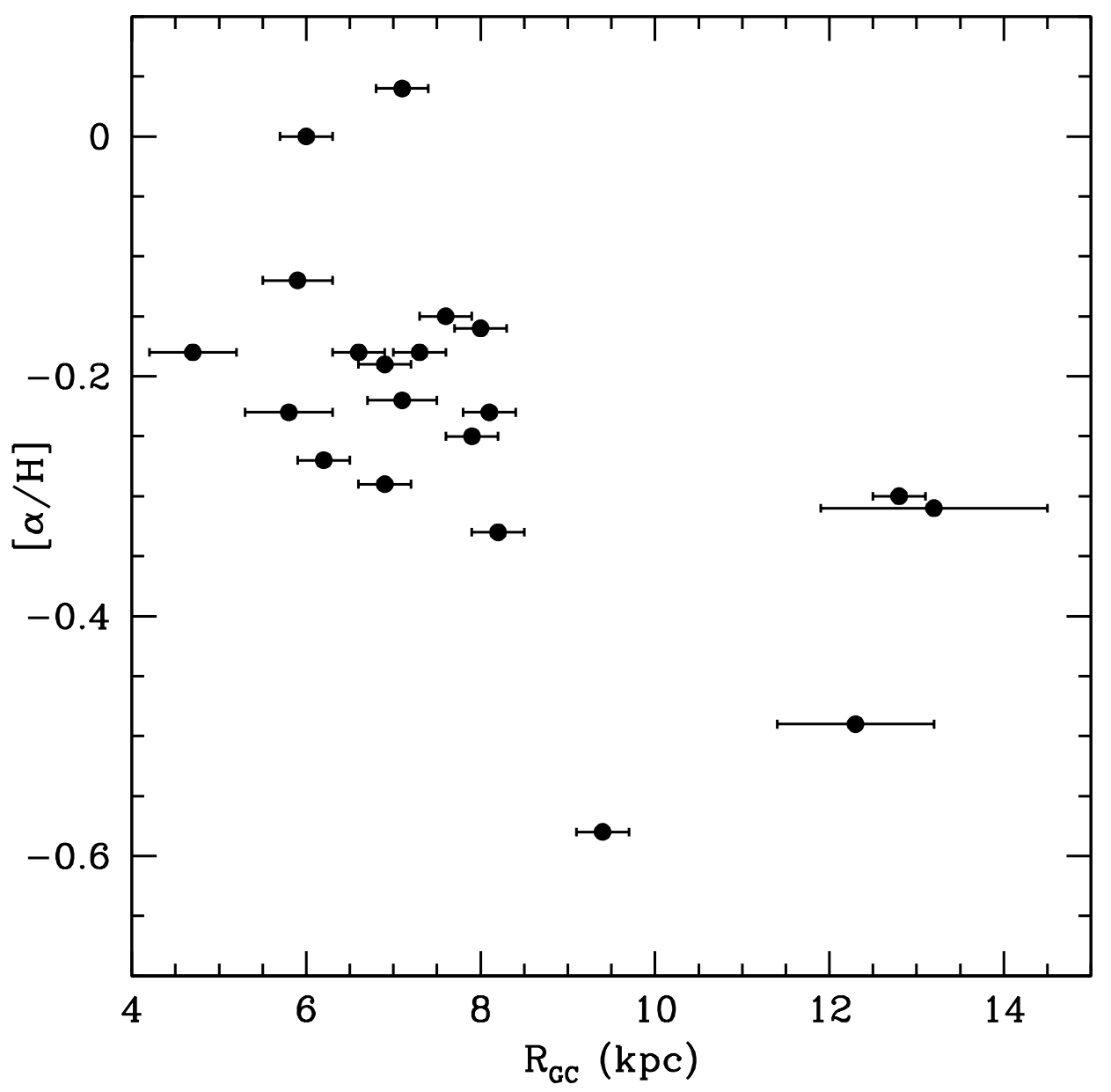

Fig. 6. - The mean values of $\mathrm{O}, \mathrm{Mg}, \mathrm{Si}$, and $\mathrm{S}$ relative to solar abundances for $\mathrm{B}$ stars studied by Daflon \& Cunha (2004). 


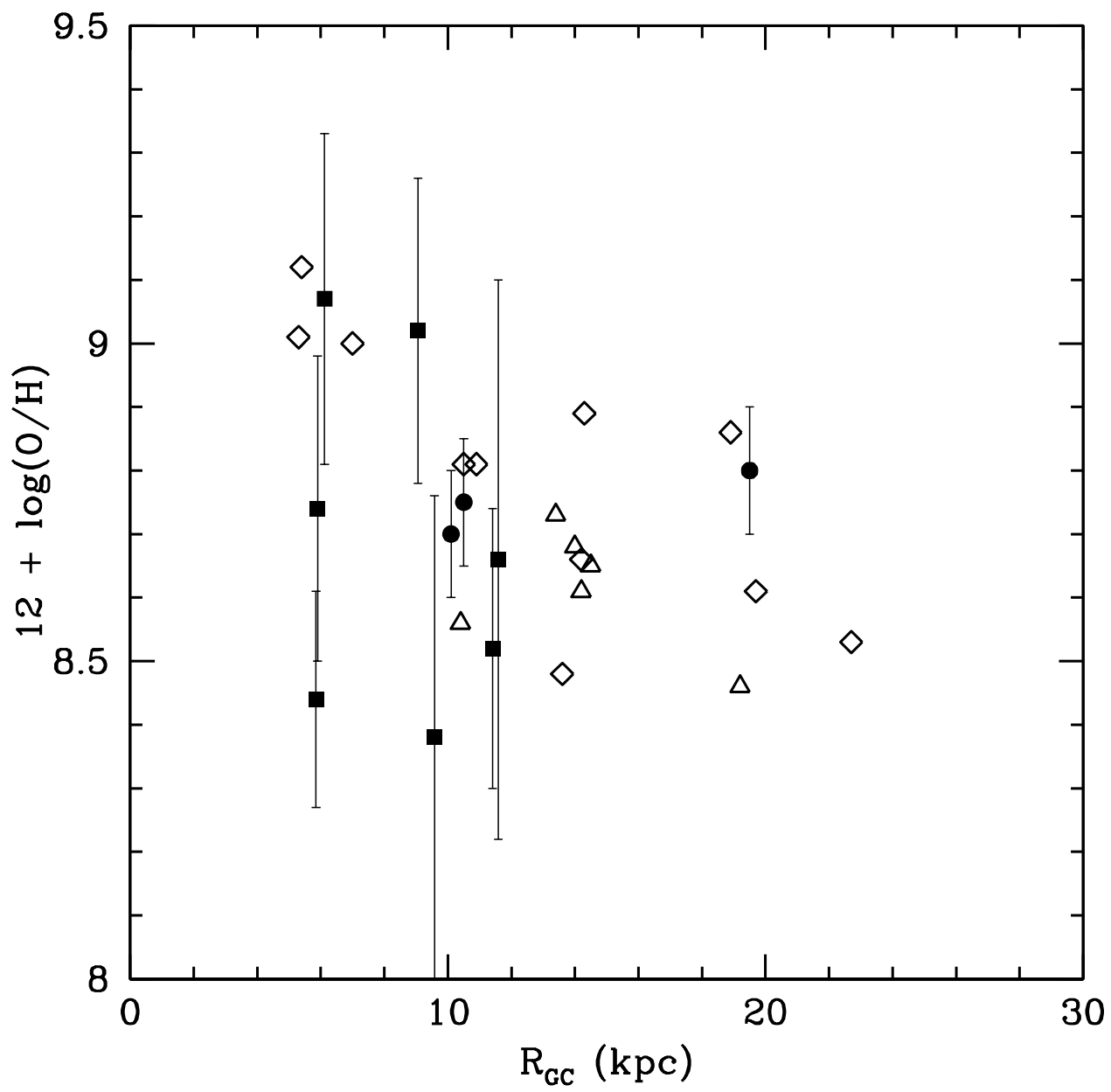

Fig. 7.- The oxygen abundances as a function of galactocentric distance in M31. Open triangles are H II regions studied by Dennefeld \& Kunth (1981), open diamonds are H II regions from Blair et al. (1982), filled circles are stars from Venn et al. (2000), and filled squares are stars from Trundle et al. (2002). 


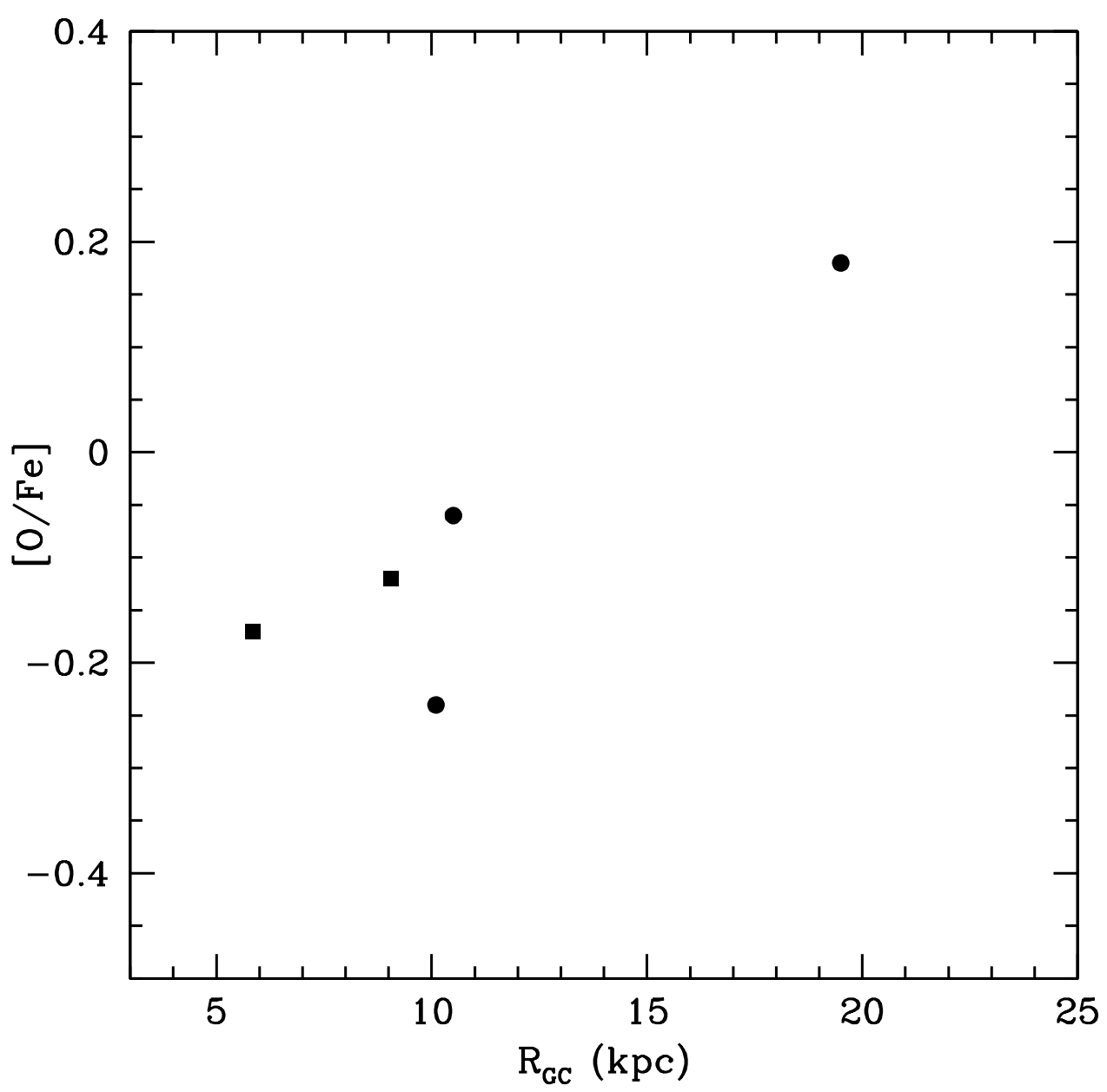

Fig. 8. - The oxygen-to-iron ratio of A and B supergiants in M31, with the same symbols as Figure 7. 
This figure "fig2.jpg" is available in "jpg" format from: http://arxiv.org/ps/astro-ph/0506210v1 